\title{
TIMING E SVILUPPO DEI SERVIZI SOCIALI IN EUROPA
}

\author{
di Valeria Fargion
}

\section{Introduzione}

La letteratura sul welfare state ha largamente privilegiato il settore pensionistico e la sanità, che peraltro costituiscono le componenti più rilevanti della spesa sociale in tutti i paesi europei. Tuttavia, nell'arco dell'ultimo decennio si è manifestato in ambito accademico un crescente interesse per le politiche socioassistenziali. La nuova attenzione nei confronti di quest'area di policy, tradizionalmente considerata del tutto marginale nel contesto dei sistemi di protezione sociale, riflette la consapevolezza che nuovi bisogni sociali vanno diffondendosi in tutta Europa. A monte dei nuovi bisogni vi sono indubbiamente i profondi cambiamenti che hanno investito le nostre società sia sotto il profilo demografico sia per quanto riguarda la struttura familiare. Tali cambiamenti stanno infatti imponendo la ricerca di nuove soluzioni per far fronte alle esigenze di cura dei soggetti che non sono in grado di provvedere autonomamente a se stessi; ciò riguarda in primo luogo la terza e la quarta età, ma anche la prima infanzia, i portatori di handicap, i malati mentali ed i malati cronici.

Se riflettiamo sulla natura degli interventi da approntare per queste nuove domande, appare evidente come - data la dimensione intrinsecamente territoriale dei servizi di care - il livello locale di governo sia quello maggiormente investito dalle problematiche emergenti. Quali sono allora, nel contesto europeo, i vincoli e le opportunità entro cui il governo locale si è trovato ad operare tra gli anni ottanta e novanta? Questo articolo si propone appunto di esplorare le condizioni strutturali che hanno rappresentato la cornice dell'attività di policy dei livelli subnazionali di governo, prestando particolare attenzione ai vincoli di natura finanziaria con cui tali soggetti hanno dovuto misurar- 
si nel più ampio contesto della crisi fiscale dello stato e delle politiche di austerità che hanno caratterizzato tutti i paesi europei a partire dagli anni ottanta.

La questione offre spunti estremamente interessanti per sviluppare anche una serie di riflessioni teoriche sull'incidenza e le implicazioni del timing nei processi di produzione delle politiche pubbliche. Nell'Europa continentale, e ancor più in quella meridionale, la tematica dei servizi sociali è emersa infatti in un contesto temporale radicalmente diverso rispetto ai paesi scandinavi, con conseguenze tutt'altro che trascurabili per quanto riguarda, ad esempio, il quadro dei vincoli macro-economici ed il ventaglio delle opzioni di policy effettivamente disponibili. Un approccio di questo tipo, che valorizza scansione e sequenze temporali dei processi di policy, può fornire una chiave interpretativa delle differenze nazionali in questa area di policy decisamente più pregnante rispetto ad un'analisi comparata di taglio esclusivamente sincronico, suggerendo l'opportunità di analoghe verifiche anche in altri campi.

\section{Cambiamenti strutturali e nuove domande}

Per inquadrare correttamente il problema conviene partire proprio da una ricognizione dei principali indicatori che consentono di delineare le dimensioni quantitative dei fenomeni in atto. Il primo di tali indicatori è sicuramente rappresentato dal tasso di invecchiamento della popolazione. Nell'arco degli ultimi quarant'anni la quota degli ultrasessantenni è costantemente cresciuta nell'ambito dell'Unione Europea, passando dal 15,3\% della popolazione complessiva rilevato nel 1960 all'attuale 20,7\% - un trend destinato nei prossimi anni a subire una ulteriore accelerazione. Secondo le stime della Commissione, intorno al 2020 l'incidenza degli anziani oscillerà infatti tra il $25 \%$ e il $29 \%$ della popolazione, con la punta più elevata in Italia. L'aumento sarà particolarmente accentuato in Finlandia, Irlanda e Paesi Bassi, che oggi presentano valori nettamente inferiori alla media. Ma forse il dato più emblematico della trasformazione demografica in corso nei paesi europei è rappresentato dal fatto che intorno al 2005 il numero delle persone con più di sessant'anni arriverà a sorpassare quello dei giovani con meno di vent'anni.

Se è vero che questo scenario è il frutto del miglioramento del tenore di vita e, se vogliamo, anche del successo dei pro- 
grammi di protezione sociale attuati dai welfare states europei, è certo che esso pone nuovi e preoccupanti problemi di policy. Gli interventi a favore degli anziani, varati soprattutto in questo secondo dopoguerra, non erano certo calibrati per ordini di grandezza così rilevanti; non solo, ma se guardiamo più attentamente le cifre possiamo constatare che la dinamica di espansione più rilevante ha riguardato e continuerà a riguardare gli ultraottantenni e cioè la fascia più esposta al rischio di perdere la autosufficienza e per cui sono di conseguenza maggiori le necessità di servizi e di assistenza. Anche se i valori assoluti restano ancora relativamente contenuti, si tratta pur sempre del $3,9 \%$ della attuale popolazione dell'Unione Europea contro $1,1,8 \%$ di solo trent'anni fa!

Tutto questo si è verificato in concomitanza con una serie di cambiamenti non meno profondi nel profilo della famiglia, segnati da un progressivo e sempre più marcato processo di deistituzionalizzazione del matrimonio, che ha portato al diffondersi lungo l'asse Nord-Sud di una varietà di modelli familiari comunque più fluidi e meno stabili. Non è questa la sede per approfondire un tema complesso come la trasformazione della famiglia occidentale, ma di nuovo conviene richiamare qualche dato. Il segnale forse più evidente della erosione del tradizionale legame coniugale è costituito dalla stabile crescita delle nascite al di fuori del matrimonio. Nel 1994 l'incidenza di tali nascite rappresentava per i quindici paesi dell'Unione il $21,7 \%$ del totale, con i principali paesi dell'Europa centro-settentrionale attestati su livelli assai superiori: oltre il $30 \%$ in Francia e Gran Bretagna e addirittura il $46 \%$ ed il $50 \%$ in Danimarca ed in Svezia, rispettivamente. In sintonia con questo quadro, l'Europa ha visto crescere la frequenza dei divorzi, delle famiglie monoparentali e delle unioni consensuali, che restano nettamente inferiori alla media europea solo nei paesi dell'area mediterranea. Il divorzio, ad esempio, è oggi l'esito di un matrimonio su quattro $^{1}$, mentre le famiglie composte da un solo genitore rappresentavano nel 1994 il 5,8\% delle famiglie (media sull'Europa a 12), con punte del (quasi) $9 \%$ in Irlanda e Gran Bretagna.

1 Secondo i dati Eurostat questo tipo di conclusione riguarda in maniera progressivamente più accentuata i matrimoni celebrati nei periodi più recenti. Infatti mentre solo il $14 \%$ dei matrimoni celebrati negli anni sessanta si è concluso o si concluderà con un divorzio, le corrispondenti stime per i matrimoni contratti nel 1970 e nel 1980 sono del 22 e del $27 \%$. 
Le difficoltà oggettive di provvedere alle funzioni di cura, connesse ad assetti familiari in cui viene meno la stabile presenza delle due figure genitoriali, si riverberano in primo luogo sull'infanzia ed a maggior ragione sugli anziani. Né le prospettive sembrano destinate a migliorare in futuro se consideriamo l'andamento delle coabitazioni al di fuori del matrimonio, che rappresentano nel complesso una situazione di maggiore precarietà rispetto alle unioni legittime. Sebbene le unioni consensuali nell'insieme siano ancora un fenomeno limitato (corrispondente al $10 \%$ circa delle coppie europee), il panorama potrebbe modificarsi rapidamente visto che tali unioni riguardano addirittura il $28 \%$ delle persone in coppia con meno di trenta anni.

Se integriamo quanto detto fin qui con alcuni riferimenti, da un lato, allo sviluppo della occupazione femminile e, dall'altro, alla evoluzione delle soluzioni abitative adottate dai nuclei familiari, appaiono inequivocabili i contorni della nuova situazione di problem pressure, che a partire dagli anni sessanta/settanta è venuta a determinarsi con intensità crescente in tutti i paesi europei. Una situazione che segue una traiettoria NordSud, partendo dall'area scandinava per diffondersi a macchia d'olio nell'Europa continentale ed attecchire progressivamente anche nell'Europa meridionale.

Non occorre spendere molte parole per ricordare quanto sia cresciuta la presenza delle donne nel mercato del lavoro, soprattutto nell'arco degli ultimi venticinque anni. Se consideriamo il tasso di attività delle donne in età compresa tra i 20 e i 59 anni, nei primi anni novanta in Europa ci troviamo di fronte ad un valore del $62 \%$ con un range che va da un minimo del $46 \%$ in Spagna ed in Grecia ad un massimo dell' $84 \%$ in Danimarca. $\grave{E}$ vero che in genere l'attività lavorativa è più elevata tra le nubili che tra le donne sposate ${ }^{2}$, ma la differenza in media è solo di 7 punti percentuali ${ }^{3}$, per cui possiamo sicuramente affermare che la condizione della donna come casalinga in grado di dedicarsi a tempo pieno a eventuali necessità di cura dei membri più deboli della famiglia rappresenta oramai un fenomeno in larga misura minoritario e destinato a contrarsi ulteriormente.

2 Nel caso del Belgio e della Francia il tasso di attività femminile risulta invece leggermente superiore tra le sposate che tra le nubili.

3 L'unica eccezione è rappresentata dal caso dell'Olanda dove la distanza è addirittura di venti punti percentuali. 
Nel sondaggio Eurostat del 1994, destinato al monitoraggio dei nuclei familiari nell'Unione ${ }^{4}$, solo il 33\% delle donne tra 25 e 59 anni si è dichiarata au foyer con percentuali di risposta più elevate esclusivamente nei quattro paesi mediterranei, in Irlanda e nei Paesi Bassi.

Un trentennio or sono sotto questo profilo il volto dell'Europa era profondamente diverso, con una marcata presenza delle donne nel mercato del lavoro confinata all'area nordica (con la parziale eccezione, in quest'area, della Norvegia, che presentava valori più bassi rispetto ai paesi limitrofi). Ma il ruolo di cura tradizionalmente affidato alle donne incontrava già allora un altro tipo di difficoltà: fatta eccezione per i paesi del Sud Europa, la famiglia estesa - caratterizzata dalla coabitazione tra più generazioni - non rappresentava più la norma ed era stata largamente sostituita dalla famiglia nucleare e dalla conseguente separazione delle abitazioni tra gli anziani ed i loro figli.

Pur mancando un quadro dettagliato della situazione, i dati statistici disponibili indicano chiaramente come, non solo nell'Europa del Nord ma anche in buona parte dell'Europa continentale, la quota di anziani che viveva con i propri figli toccasse già alla fine degli anni sessanta appena il 20/25\% del totale. Perfino in un paese come l'Olanda, con un forte insediamento sub-culturale cattolico, la stragrande maggioranza degli anziani viveva già allora in abitazioni distinte. Nei due decenni successivi il declino della famiglia estesa ha subito una ulteriore accelerazione, cosicché - se escludiamo la Spagna ${ }^{5}$, l'Italia e l'Irlanda - la coabitazione pare ormai vicina a scomparire del tutto. Non desta quindi sorpresa che nella documentazione statistica dell'Unione la condivisione dell'alloggio tra anziani e figli adulti ricada sotto la categoria ménage atipique!

L'intreccio dei fattori che abbiamo preso in considerazione riduce oggettivamente le possibilità di cooperazione inter-generazionale, accentuando la difficoltà di provvedere alle funzioni di accudimento entro la rete sociale primaria. Per di più, l'insieme dei problemi che scaturiscono dall'interazione di questi

4 Si tratta del Panel Communautaire de Ménages, la cui prima tornata è stata realizzata appunto nel 1994. Si confronti in proposito Eurostat, Statistiques en bref. Population et conditions sociales, 1997, n. 5 .

5 In base a dati statistici più recenti, il tasso di coabitazione in Spagna si collocherebbe attualmente intorno al $24 \%$, il che suggerisce che lo scenario potrebbe cambiare assai rapidamente anche nell'Europa meridionale. 
cambiamenti demografici, occupazionali e della famiglia è destinato ad aggravarsi ulteriormente nel prossimo futuro. A questo proposito le proiezioni demografiche elaborate dalla Commissione Europea (1997) illustrano puntualmente la misura in cui l'invecchiamento della popolazione inciderà sulle singole realtà europee. Basti dire che mentre a metà degli anni novanta i cittadini sopra i sessantacinque anni superavano il $20 \%$ della popolazione solo in alcune regioni europee - notoriamente nell'Italia centrale - nel 2015 questa sarà la situazione dominante in tutta Europa.

\section{Lo sviluppo precoce dei servizi sociali nell'area scandinava}

Come accennato nell'introduzione, i cambiamenti su cui abbiamo focalizzato l'attenzione esercitano pressione soprattutto sul livello di governo locale, in ragione della intrinseca dimensione territoriale dei servizi sociali. Merita subito aggiungere che sono state le autorità locali dei paesi nordici a muoversi per prime su questo terreno e con largo anticipo rispetto al resto dell'Europa. Già tra gli anni sessanta e settanta in Scandinavia il governo locale aveva infatti dato vita ad una rete capillare di servizi sociali pubblici; al punto che riferendosi all'area nordica, Sipilä si domanda se non dovremmo piuttosto parlare di welfare municipalities anziché di welfare states $(1997,7)^{6}$.

Mettere a fuoco le peculiarità dell'esperienza scandinava può a mio avviso fornire utili chiavi di lettura per inquadrare il diverso percorso seguito dagli altri paesi europei. Alla luce di questo intento, nonostante l'interesse prevalente degli studiosi per le differenze tra i singoli casi, mi concentrerò sui tratti comuni all'insieme dei paesi nordici. Più precisamente, per comprendere a pieno il corso d'azione seguito dagli attori di policy nell'ambito scandinavo, prenderò in considerazione una serie di fattori: il contesto temporale in cui si sono manifestati i nuovi bisogni sociali; la mediazione culturale che ne ha favorito la legittimazione; gli assetti istituzionali entro cui si è sviluppato il processo di policy.

Abbiamo già ricordato come in Scandinavia i cambiamenti

6 Come la letteratura ha ampiamente dimostrato, ciò che caratterizza il modello di servizi sociali scandinavo è l'ampiezza dei bisogni sociali coperti e l'universalità dell'accesso. 
demografici $e$ - in misura ancora più marcata - i cambiamenti occupazionali e della famiglia si siano verificati in una fase precedente rispetto al resto dell'Europa. All'inizio degli anni settanta in Svezia ed in Danimarca il 67\% delle donne in età lavorativa era impegnato nel mercato del lavoro, mentre in Finlandia la percentuale era solo di qualche punto inferiore. Ma questo non può che rappresentare il primo passo del nostro discorso. La tappa successiva consiste nel considerare come le nuove potenziali domande abbiano interagito con il retroterra culturale. A questo proposito vale la pena di ricordare che limitando la loro analisi a fattori di natura strettamente strutturale, Antonnen e Sipilä $(1996,93)$ non sono in grado di spiegare perché - nonostante livelli analoghi di occupazione femminile - Francia e Germania mostrino un livello così diverso di servizi per l'infanzia.

Il retroterra culturale. I mutamenti sociali che ci interessano, pur avendo implicazioni per un'ampia gamma di valori, incidono soprattutto sul modo in cui viene concepito il ruolo della famiglia e della donna al suo interno. In particolare possiamo ipotizzare che la problematica dei servizi sociali entri nell'agenda politica in momenti storici diversi, a seconda del diverso grado di radicamento della visione tradizionale delle responsabilità femminili. In altri termini, l'eredità culturale di un dato paese può favorire, o al contrario ostacolare, il processo di legittimazione relativo alla esternalizzazione delle funzioni di care tradizionalmente confinate entro l'ambito familiare.

In altra sede (Fargion 1997a) ho proposto di definire questo processo come «defamilizzazione» delle funzioni di care. Se è vero che anche altri autori hanno fatto riferimento a questo concetto, sia pur utilizzando una terminologia leggermente diversa, mi sembra che sia possibile farne uno strumento analitico più fruttuoso rispetto all'uso corrente. Più precisamente, la «defamilizzazione» può essere collocata lungo un continuum in cui le specifiche funzioni di care che vengono esternalizzate corrispondono a tappe progressivamente più dirompenti rispetto alla lettura tradizionale delle responsabilità istituzionali della famiglia. In questa prospettiva la proiezione all'esterno della struttura familiare di funzioni attinenti l'assistenza agli anziani costituisce il polo di partenza di un percorso che culmina con la legittimazione di forme di accudimento extra-familiare nei confronti della prima infanzia. Appare infatti straordinariamente ostico aggredire il nucleo fondante dell'istituto familiare che 
ruota intorno alla riproduzione e all'allevamento dei figli: funzioni radicatamente connesse nel panorama occidentale alla donna nel suo ruolo di madre (Windebank 1996) ${ }^{7}$.

Date queste premesse, Svezia, Danimarca, Finlandia e, se pur in misura minore, Norvegia appaiono come i paesi più inclini e culturalmente più predisposti a recepire il processo di «defamilizzazione» nella sua accezione più estensiva. A questo proposito appare particolarmente illuminante una recente interpretazione dell'ethos che caratterizzerebbe il modello nordico di protezione sociale, decisamente contro-corrente rispetto all'immagine stereotipata che siamo abituati ad incontrare nella letteratura.

Nonostante la percezione comune della natura collettivistica del welfare state nordico, ciò che è ancor più sorprendente è la misura in cui dietro la $\mathrm{Ge}$ meinschaft della cosiddetta «casa della gente» si trovi una Gesellschaft di individui autonomi e atomizzati [...] il welfare state nordico e in particolare quello svedese può essere visto come un gigantesco patto attraverso cui gli individui si sono collettivamente affrancati dalle responsabilità personali, individuali sotto la veste di una solidarietà gestita dallo stato (Trägårdh 1997, 253, 262).

Secondo questa lettura, il taglio individualistico rintracciabile nelle politiche della famiglia sviluppate soprattutto dal 1970 in poi non sarebbe che l'ultimo anello di una lunga catena che affonda le radici nel passato delle popolazioni nordiche e paradossalmente nella loro asocialità ed insofferenza per qualsiasi legame che costituisca una forma di dipendenza.

Sia che si condivida questa chiave di lettura sia che si preferisca l'immagine di una società amica delle donne, resta il fatto che le lenti culturali attraverso cui sono stati filtrati i nuovi bisogni non hanno certo costituito una barriera alla «defamilizzazione». Al contrario, il background culturale dei paesi nordici ha rappresentato un elemento propulsore nei confronti dell'intervento pubblico visto come mezzo non solo per alleviare il peso delle funzioni di accudimento tradizionalmente assegnate alle donne, ma anche per promuovere un'effettiva uguaglianza di opportunità tanto nel mercato del lavoro quanto nella società nel suo complesso. Del resto le disposizioni di legge in vigore

7 Rispetto a quest'ultima ipotesi, la «defamilizzazione» di funzioni di care nei confronti di soggetti con problemi di handicap o di devianza si configura in termini meno traumatici perché i nuovi orizzonti aperti dalla evoluzione della riflessione scientifica in materia delineano una potenziale «incompetenza» della famiglia sotto il profilo medicoterapeutico e riabilitativo. 
nei paesi scandinavi testimoniano come la titolarità dei diritti sociali sia passata dalla famiglia all'individuo assai prima rispetto a tutti gli altri paesi europei. In Svezia, ad esempio, fin dal 1956 il mantenimento degli anziani non è stato più considerato come un obbligo a carico dei figli; lo stesso è avvenuto in Norvegia nel 1964 ed in Finlandia nel 1970, mentre in Danimarca questo obbligo non era mai stato sancito a livello normativo.

Un governo locale forte. Gli elementi che abbiamo fin qui richiamato possono aiutarci ad inquadrare solo le pre-condizioni fattuali e valoriali con cui hanno dovuto misurarsi i policy makers, ma per capire come e perché i paesi nordici abbiano creato una rete di servizi sociali così estesa e capillare non possiamo prescindere dal fatto che essi hanno potuto contare su un sistema di governo locale particolarmente forte, soprattutto in prospettiva comparata.

Già negli anni cinquanta gli enti locali avevano accumulato una notevole esperienza di gestione dei servizi pubblici nel settore dell'istruzione, della casa e dell'assistenza sanitaria ed ospedaliera. Lungi dal costituire una novità, l'acquisizione di nuove competenze nella sfera sociale poteva quindi essere interpretata agevolmente come un ampliamento dei precedenti impegni a favore del benessere della popolazione. Inoltre, a seguito delle riforme degli anni cinquanta e sessanta, ed in particolare della ristrutturazione territoriale dei comuni (Page 1987), questi ultimi avevano acquisito capacità strutturali ed organizzative tali da consentire una gestione efficace ed efficiente dei nuovi compiti. Grazie alla forte riduzione del numero dei comuni derivante dai processi di fusione, le nuove municipalità potevano contare su dimensioni territoriali in grado di consentire economie di scala, superando le difficoltà finanziarie ed operative connesse alla precedente frammentazione amministrativa.

$\mathrm{Ma}$ ancor più importante è l'autonomia fiscale di cui godeva il governo locale nell'area scandinava. All'inizio degli anni sessanta - come del resto ancor oggi - gli enti locali disponevano di una capacità impositiva nettamente superiore rispetto a tutti gli altri paesi europei. Se consideriamo tuttavia il ruolo trainante esercitato dalla legislazione nazionale nella espansione dei servizi sociali scandinavi, il nostro richiamo alla autonomia finanziaria delle autorità locali può apparire a prima vista fuori luogo. Il governo locale scandinavo è infatti spesso descritto come «una efficiente macchina attuativa delle politiche decise a 
TAB. 1 Spesa locale in percentuale del Pil e della spesa pubblica totale, e imposte dirette locali in percentuale del Pil in nove paesi europei (valori medi 1960-1964)

\begin{tabular}{lccc}
\hline & Spesa locale in \% Pil & $\begin{array}{c}\text { Spesa locale in \% } \\
\text { su spesa pubblica totale }\end{array}$ & $\begin{array}{c}\text { Imposte dirette locali in } \\
\% \text { Pil }\end{array}$ \\
\hline Svezia & 13,7 & 35,9 & 5,3 \\
Norvegia & 12,9 & 36,8 & 8,4 \\
Danimarca & 8,4 & 30,1 & 5,4 \\
Olanda & 13,2 & 28,2 & 0,3 \\
Gran Bretagna & 10,3 & 25,4 & 3,1 \\
Irlanda & 9,9 & 25,6 & 3,0 \\
Germania & 7,8 & 23,4 & 3,1 \\
Francia & 6,9 & 23,6 & 2,8 \\
Italia & 7,9 & 26,1 & 2,6 \\
\hline
\end{tabular}

Fonte: Council of Europe (1975)

livello nazionale» ovvero come «una agenzia di erogazione di servizi priva di autonomia» (Kröger 1997, 97). In contrasto con tali caratterizzazioni, ritengo che sia difficile spiegare la capillarità del sistema di servizi sociali messo in piedi nei paesi scandinavi senza fare riferimento al contributo decisivo fornito dalle istituzioni locali sia in termini di legittimazione che di sostegno finanziario. Prova ne sia che in tutta l'area l'autonomia impositiva si salda strettamente alla immagine popolare del governo locale come primo e immediato canale di partecipazione democratica finalizzato al soddisfacimento dei bisogni dei cittadini. Non a caso il diritto da parte delle autorità locali di imporre proprie tasse è considerato elemento fondante non solo dell'autonomia locale ma della stessa democrazia. Con questo non intendo sottovalutare il ruolo esercitato sotto il profilo regolativo dal livello centrale di governo, in particolare per quanto riguarda la definizione di standard uniformi di servizio. Non si tratta certo di contrapporre il livello locale a quello nazionale, tanto più che entrambi hanno condiviso a lungo analoghe maggioranze a guida socialdemocratica. In effetti per un considerevole arco di tempo questo è stato probabilmente il più efficace meccanismo di coordinamento nell'ambito dei rapporti centro-periferia. Quello che vorrei sostenere è semplicemente che occorre prestare maggior attenzione all'autonomia fiscale del governo locale come fattore cruciale nello sviluppo del modello scandinavo rispetto a quanto emerge dalla letteratura.

I dati riportati nella tabella 1 documentano eloquentemente 
TAB. 2. Tipologia del governo locale in Europa (dati relativi agli anni sessanta)

\begin{tabular}{lcccc}
\hline & \multicolumn{4}{c}{ Spesa locale } \\
\cline { 2 - 5 } & Alta & Media & Bassa \\
\hline \multirow{2}{*}{ Tassazione locale } & Sedia & Norvegia & Danimarca & \\
& Bassa & Olanda & $\begin{array}{c}\text { Gran Bretagna } \\
\text { Irlanda }\end{array}$ & $\begin{array}{c}\text { Germania } \\
\text { Media }\end{array}$ \\
& & & $\begin{array}{c}\text { Francia } \\
\text { Italia }\end{array}$ \\
\hline
\end{tabular}

come nella fase di avvio del sistema di servizi sociali scandinavo le condizioni finanziarie del livello locale differissero dal resto dei paesi europei. Le relative informazioni sono tratte da uno studio del Consiglio d'Europa (Council of Europe 1975) e si riferiscono all'incidenza media (per gli anni 1960-1964) della spesa e della tassazione locale rispetto al prodotto interno e alla spesa pubblica totale in nove paesi europei. Per rendere ancora più esplicito il discorso abbiamo incrociato le risultanze della prima e della terza colonna, che hanno come parametro di riferimento omogeneo il prodotto interno lordo (cfr. tab. 2). Tale incrocio ci permette di identificare combinazioni diverse di spesa e di prelievo delle risorse nazionali da parte del livello locale di governo. Come si può facilmente constatare, mentre nell'area nordica gli enti locali all'inizio degli anni sessanta avevano già consolidato una tradizione di intervento finanziariamente rilevante cui si abbinava una consistente capacità impositiva, troviamo una realtà diametralmente opposta in Francia e in Italia, dove la bassa incidenza delle risorse spese localmente va di pari passo con una scarsa autonomia finanziaria. Rispetto a questi due poli Gran Bretagna ed Irlanda si collocano in una posizione intermedia, analoga a quella della Germania per quanto riguarda le fonti di finanziamento, ma non per le attività di spesa, che risultano considerevolmente più ridotte in quest'ultimo paese. Lo scompenso più vistoso si ha comunque in Olanda, dove un livello di spesa praticamente uguale alla Svezia e alla Norvegia si coniuga con una dipendenza pressoché assoluta dai trasferimenti del centro. Alla luce di queste risultanze appare evidente come nell'area scandinava gli enti locali si trovassero in condizioni incomparabilmente migliori rispetto al resto d'Europa per affrontare le nuove domande. 
Implicazioni ed effetti del timing. In questo paragrafo ho fatto riferimento, sia pur genericamente, alla combinazione favorevole che caratterizza il contesto scandinavo per quanto riguarda l'emergere di nuove domande sociali, il background culturale e l'assetto istituzionale del governo locale. Tuttavia, per valorizzare pienamente gli elementi fin qui forniti, occorre fare un passo ulteriore. Ciò che appare cruciale è il momento temporale e cioè esattamente quando si viene a determinare una interconnessione tra $\mathrm{i}$ tre processi considerati. Come sostiene Pierson $(1999,16)$ : «il quando dell'emergere di una specifica questione o conflitto in una società può essere cruciale per due motivi: da un lato, il repertorio delle risposte possibili è storicamente determinato; dall'altro, una volta adottata una risposta, essa può generare dinamiche autorafforzantesi, le quali posizionano la politica su un determinato percorso di lungo termine».

Entrambi i punti sollevati dall'autore appaiono rilevanti nel nostro caso. Cominciando dal primo, indubbiamente rivestono un'importanza determinante le condizioni macro-economiche che contraddistinguono lo specifico contesto storico che ci interessa. Ma quali erano queste condizioni nell'area nordica? In quello che è forse il più ampio ed organico studio sui servizi sociali scandinavi, Sipilä $(1997,3)$ ci offre una risposta inequivocabile: «dal momento che si era deciso che tutte le madri e le figlie che ne avessero bisogno dovevano poter contare su servizi sociali di supporto, occorreva dare il via ad una produzione di servizi senza precedenti. Questo è esattamente quello che è successo in tutti i paesi scandinavi dall'inizio degli anni sessanta in poi, in una situazione in cui l'economia non poneva alcun limite alla crescita del welfare state». Come è noto, in questa fase siamo ancora nell'ambito del boom economico post-bellico e la costruzione del modello sociale scandinavo ha quindi potuto sfruttare le opportunità finanziarie derivanti da un contesto di espansione economica e dal clima ottimistico che lo accompagnava sia in ambito sociale che politico. Dal momento che la torta cresceva i decisori pubblici non erano costretti a misurarsi con vincoli di natura fiscale e potevano scegliere tra una gamma più vasta di alternative di policy rispetto a quanto sarebbe invece avvenuto nei due successivi decenni, segnati dalla crisi fiscale dello stato e da un clima di austerità.

Questa impostazione appare particolarmente utile per una riflessione comparata sui servizi sociali in Europa. Per gettare luce sulle differenze internazionali relative allo sviluppo di que- 
st'area di policy, occorre tenere presente che il modello scandinavo di social care è stato messo in piedi prima che la recessione economica e la crisi fiscale si diffondessero nei paesi occidentali. Possiamo quindi attenderci percorsi diversi nei singoli paesi a seconda delle opportunità economiche presenti nella specifica congiuntura storica in cui la problematica dei servizi sociali è stata posta all'ordine del giorno.

Sarebbe tuttavia riduttivo limitare il discorso alla configurazione delle variabili economiche nel momento in cui si intersecano le traiettorie relative ai processi rilevanti per la tematica dei servizi di social care. Ancora una volta i paesi scandinavi possono fornirci spunti interessanti. Se infatti estendiamo l'orizzonte temporale della nostra analisi, ci troviamo di fronte ad una sorta di meccanismo a senso unico per cui l'espansione dei servizi sociali avviata negli anni sessanta e settanta è proseguita ben oltre la fine del boom economico. In altri termini, assumendo un'ottica di lungo periodo possiamo agevolmente riscontrare come le misure di policy adottate nei periodi successivi in tutti e quattro i paesi scandinavi riflettano una dinamica sostanzialmente auto-rinforzante.

Come si evince dall'ampia riflessione teorica sulla path dependency, l'elemento cruciale è dato da un feedback loop di segno positivo, il quale - una volta che si siano compiuti i passi iniziali in una particolare direzione - fa sì che i passi successivi proseguano lungo lo stesso cammino. Sebbene questo non sia assolutamente un risultato scontato ed inevitabile, è certo che le politiche sociali sono state spesso identificate come un'area privilegiata per lo sviluppo di processi path dependent. Nel nostro caso del resto possiamo facilmente elencare una serie di fattori in grado di favorire effetti di chiusura (i cosiddetti lock-in effects). In particolare, non possiamo non considerare che eventuali ritorni all'indietro avrebbero non solo dovuto scontrarsi con l'opposizione di un numero estremamente vasto di beneficiari ma anche con i radicati interessi di una consistente quota della forza lavoro direttamente impegnata nella erogazione dei servizi stessi.

Inoltre anche quando lo scenario economico ha cominciato progressivamente a peggiorare, l'elevata autonomia impositiva ha consentito agli enti locali di godere di un margine di manovra più consistente nella contrattazione con il livello nazionale di governo. Tornerò più ampiamente su questo punto nel paragrafo finale; per il momento mi preme sottolineare che, in fun- 
zione di pervasivi meccanismi inerziali e di una serie di conseguenze inattese derivanti da misure adottate in precedenza, la distanza tra i paesi scandinavi e l'Europa continentale è addirittura aumentata via via che essi procedevano lungo il cammino imboccato all'inizio degli anni sessanta.

\section{Il ritardo temporale dell'Europa continentale e mediterranea}

Se a questo punto volgiamo lo sguardo a quanto accadeva tra gli anni sessanta e settanta negli altri paesi europei, non è difficile rilevare come a livello sociale le spinte per una riduzione delle funzioni di accudimento proprie della famiglia fossero ancora tutto sommato assai contenute rispetto all'esperienza dei paesi nordici. A questo proposito non si può ignorare che nel complesso il tasso di occupazione femminile era notevolmente inferiore rispetto a quello scandinavo. Ciò non toglie che in alcuni paesi - ed in particolare in Francia, Germania e Gran Bretagna - la partecipazione delle donne al mercato del lavoro fosse tutt'altro che trascurabile. D'altro canto anche il passaggio dalla famiglia estesa alla famiglia nucleare costituiva già un fenomeno largamente diffuso e consolidato in buona parte dell'Europa continentale; il che rendeva tutt'altro che remota l'esigenza di mettere in piedi una rete di servizi territoriali per gli anziani. In sintesi, i cambiamenti di natura demografica, occupazionale e familiare - che hanno costituito l'elemento trainante dei processi di «defamilizzazione» nell'area scandinava - erano già in corso in numerosi altri paesi europei, ma non avevano ancora raggiunto una soglia critica.

Nella maggior parte dell'Europa continentale - per non parlare di quella mediterranea - il retroterra culturale costituiva inoltre un formidabile ostacolo a qualsiasi tentativo di spostare, sia pur in parte, il peso delle funzioni di accudimento dalla sfera familiare a quella pubblica. A questo proposito è decisamente emblematico il caso della Germania. In questo paese ci troviamo infatti di fronte ad una sorta di ipostatizzazione della famiglia coniugale che, sorretta dalla rigidità e dal formalismo dei dispositivi normativi, ha teso a congelarne l'immagine convenzionale con tutto ciò che l'accompagnava in termini di obbligazioni morali e materiali. Non dobbiamo dimenticare che la famiglia coniugale è formalmente richiamata nella costituzione tedesca e che nei suoi confronti è prevista una protezione privile- 
giata da parte dello stato. Come utilmente sottolinea Schulteis: «Questa consacrazione statale di un particolare modello familiare, che esattamente come nel caso del principio di sussidiarietà della politica sociale federale è oggetto di un controllo vigilante da parte della Corte costituzionale, si è tradotta in un elemento frenante non trascurabile rispetto ai tentativi di "modernizzazione" del diritto civile e sociale della famiglia» (1996, 218). Non a caso gli unici servizi sociali che in Germania hanno ricevuto una qualche attenzione politica ed un sostegno finanziario pubblico sono quelli destinati agli anziani, che come ho suggerito più sopra riflettono solo lo stadio iniziale e meno controverso del processo di «defamilizzazione».

Ciò nonostante nell'arco degli anni ottanta, e ancor più negli anni novanta, perfino in paesi caratterizzati da un contesto culturale particolarmente sfavorevole come la Germania e l'Olanda, l'intero intreccio delle questioni connesse alle esigenze di assistenza è venuto inesorabilmente alla ribalta e la issue dei servizi sociali ha fatto il suo ingresso nell'agenda politica. Via via che i mutamenti sociali illustrati nel primo paragrafo si propagavano lungo l'asse Nord-Sud e venivano filtrati da lenti culturali più favorevoli grazie alla sempre più marcata diffusione di processi di secolarizzazione, la tematica dei servizi sociali ha acquisito una crescente visibilità politica non solo nell'Europa continentale, ma perfino in alcuni paesi dell'area mediterranea.

Lo scarto temporale rispetto ai paesi nordici ha però fatto sì che il resto dell'Europa si trovasse ad affrontare la questione dei servizi sociali in condizioni nettamente diverse rispetto ai suoi precursori. Basti pensare alla diversità del contesto macroeconomico: al clima espansionistico tipico della fase precedente, è subentrato dagli anni ottanta in poi un clima di austerità che si è tradotto innanzitutto in politiche di contenimento della spesa pubblica, in un quadro internazionale caratterizzato dalla liberalizzazione dei mercati e dalla globalizzazione della competizione. È sullo sfondo di questo scenario che dobbiamo considerare le politiche dei singoli paesi nel settore che qui ci interessa.

Per cogliere a pieno le implicazioni del nuovo contesto, dobbiamo tornare a focalizzare l'attenzione sul peso e la forza del governo locale nei singoli paesi (Alber 1995). Dal momento che il variegato insieme di domande che scaturiscono dal processo di «defamilizzazione» incide prima e soprattutto sul livello locale di governo, è particolarmente rilevante mettere in luce 
il quadro dei vincoli e delle opportunità entro cui si è mossa l'azione locale in questo specifico arco di tempo.

Da un capo all'altro dell'Europa questo periodo è segnato $\mathrm{da}$ un rinnovato interesse per le politiche di decentramento. Se a prima vista ciò può sembrare positivo, ad un'analisi più approfondita ci accorgiamo che le cose non stanno esattamente così. Mentre nei precedenti decenni la devoluzione di poteri dal centro alla periferia rispondeva ad esigenze di tipo funzionale (Dente e Kjellberg 1988), tra gli anni ottanta e novanta l'asse portante delle nuove politiche di decentramento riflette in primo luogo il riorientamento di mercato derivante dal predominio incontrastato dei valori neo-liberali. Ed è interessante notare che tanto la variante di destra che quella di sinistra del neoliberalismo sostengono l'opportunità e l'utilità di un più ampio decentramento. Mentre da sinistra si sottolinea come il rafforzamento delle competenze locali può consentire una migliore capacità di risposta della sfera pubblica ai bisogni dei cittadini, da destra le argomentazioni a favore delle politiche di decentramento fanno soprattutto riferimento a concetti quali riduzione di costi, libertà di scelta, responsabilità individuale, competizione fiscale e sussidiarietà.

$\mathrm{Ma}$ in che modo tutto ciò incide sulla politica dei servizi sociali e sulle sue prospettive future? Secondo Bennett $(1993,15)$, la nuova impostazione - «favorendo un approccio più orientato al consumatore e l'utilizzazione di strutture di mercato» - sta facendo emergere a livello di governo locale «un modello postwelfare» con implicazioni diverse a seconda di quello che è il punto di partenza in ciascun sistema. I paesi del Sud Europa, ad esempio, «potrebbero saltare in larga misura la fase della erogazione diretta per passare direttamente a modalità di gestione dei servizi parastatali, quasi-statali e di mercato».

Se è vero che è necessario un impegno di ricerca di ben più vasto raggio per avere un quadro esauriente di ciò che sta avvenendo, possiamo comunque trarre elementi di riflessione estremamente utili da un'analisi delle tendenze in atto nei rapporti centro-periferia in Europa; ciò per inquadrare correttamente l'evoluzione delle politiche non solo nel campo in esame, ma anche in altri settori che stanno acquisendo una rilevanza crescente, quali l'assistenza pubblica e le politiche attive del lavoro. Nonostante la diversità degli assetti istituzionali vigenti nei diversi paesi, i livelli sub-nazionali di governo si trovano infatti sempre più coinvolti nel sostenere il peso finanziario dei pro- 
grammi di assistenza pubblica - vedi il caso della Francia, della Germania e del Belgio, per non parlare della Spagna e dell'Ita$\mathrm{lia}^{8}$, dove fino ad oggi gli unici interventi di questo tipo erano gestiti a livello regionale e locale. Considerazioni di taglio analogo possono essere espresse sulle misure destinate al reinserimento dei disoccupati nella forza lavoro attiva. A prescindere dalla diversa propensione nazionale per il workfare inglese o per l'insertion social di matrice francese, le politiche volte a combattere la disoccupazione e l'esclusione sociale ricadono sempre più nell'ambito di competenza del livello locale, richiedendo un investimento significativo di risorse locali.

$\mathrm{Ma}$ come possono i livelli sub-nazionali di governo affrontare le nuove sfide in un contesto in cui è sostanzialmente da escludere un ulteriore innalzamento della pressione fiscale? In questo quadro, il nodo cruciale riguarda chiaramente come sono distribuite le risorse tra i diversi livelli di governo.

\section{La spesa locale in un quadro di vincoli di bilancio}

La sostanza della ipotesi che sto cercando di sviluppare è che, dato il diverso timing con cui la questione dei servizi sociali è stata posta all'ordine del giorno nei paesi dell'Europa continentale e mediterranea, tali paesi non erano assolutamente in condizione di seguire un percorso analogo a quello dei paesi scandinavi, e di creare cioè una rete territoriale di servizi sociali pubblici. Nell'affrontare il tema dei servizi di care, i decisori pubblici erano in larga misura obbligati a seguire un'impostazione che consentisse di valorizzare un'ampia gamma di soggetti non pubblici. Così facendo, essi erano infatti in grado di ampliare sia le fonti di finanziamento che la gamma dei fornitori. All'interno di queste coordinate generali, gli esiti nazionali sono ovviamente risultati diversi a seconda del ventaglio di opzioni disponibili nei singoli contesti. È evidente come il ristretto numero delle potenti organizzazioni confessionali tedesche operanti nel terzo settore abbia ben poco a che vedere con il variegato mondo delle cooperative e delle associazioni non profit cresciuto in Francia soprattutto a partire dagli anni ottanta.

8 Come noto, un programma di reddito minimo è stato recentemente introdotto in Italia, ma per il momento esso ha carattere solo sperimentale e coinvolge un piccolissimo numero di comuni. 
Un buon punto di partenza per sviluppare questa linea di ragionamento può essere fornito dall'andamento complessivo dei livelli di spesa da parte delle autorità locali nell'arco di tempo che stiamo considerando. A questo proposito è possibile ad esempio individuare una qualche tendenza comune sottostante le singole traiettorie nazionali? $\mathrm{Ci}$ troviamo cioè di fronte a trends di aumento o diminuzione nei livelli di spesa locale largamente condivisi dall'insieme di questi paesi o abbiamo a che fare con percorsi nazionali divergenti e sostanzialmente riconducibili solo alla specificità dei singoli casi? Le statistiche elaborate dall'Ocse rappresentano ai nostri fini un'ottima fonte di informazione, anche se i valori non sono purtroppo direttamente comparabili con quelli riportati nella tabella 1 , tratti come si è detto da uno studio del Consiglio d'Europa.

Nella figura 1 troviamo dati relativi a sei paesi europei: Gran Bretagna, Germania, Francia, Italia, Olanda e Belgio. La figura fornisce una prima risposta ai nostri interrogativi, illustrando la variazione cumulativa annuale nella spesa locale dei sei paesi ed il contributo specifico fornito da ciascuno di essi9. Più precisamente, documentando i cambiamenti percentuali di spesa rispetto all'anno precedente per il periodo 1985-1996, la figura fornisce una illustrazione grafica della variazione complessiva nell'insieme dei sei paesi, della direzione di tale variazione, e dell'andamento relativo a ciascun paese.

Come possiamo rilevare, passando dalla metà degli anni ottanta alla metà degli anni novanta la variazione totale del volume di spesa locale diminuisce sensibilmente e nell'insieme si manifesta in maniera sempre più nitida un andamento decrescente. Se all'inizio del periodo sembra esserci ancora spazio per un aumento - sia pur limitato - dell'intervento locale, negli anni novanta ci troviamo di fronte ad un sostanziale congelamento dei livelli di spesa con aggiustamenti in più $o$ in meno del tutto marginali. Date queste coordinate di fondo, possiamo a questo punto impostare un'analisi più ravvicinata dei singoli casi nazionali. Per limiti di spazio, concentrerò l'attenzione sul-

9 Come passo preliminare ho considerato i livelli assoluti di spesa locale nei singoli paesi e ho quindi convertito i valori correnti in valori costanti, utilizzando come parametro di riferimento l'indice dei prezzi per i consumi pubblici finali a base 1990 . Tuttavia, ciò che risulta saliente per sviluppare la mia riflessione sul timing non è il valore assoluto della spesa locale, ma piuttosto se ed in che misura la capacità di spesa del governo locale stesse crescendo nella fase in cui la tematica dei servizi sociali è entrata in agenda. 


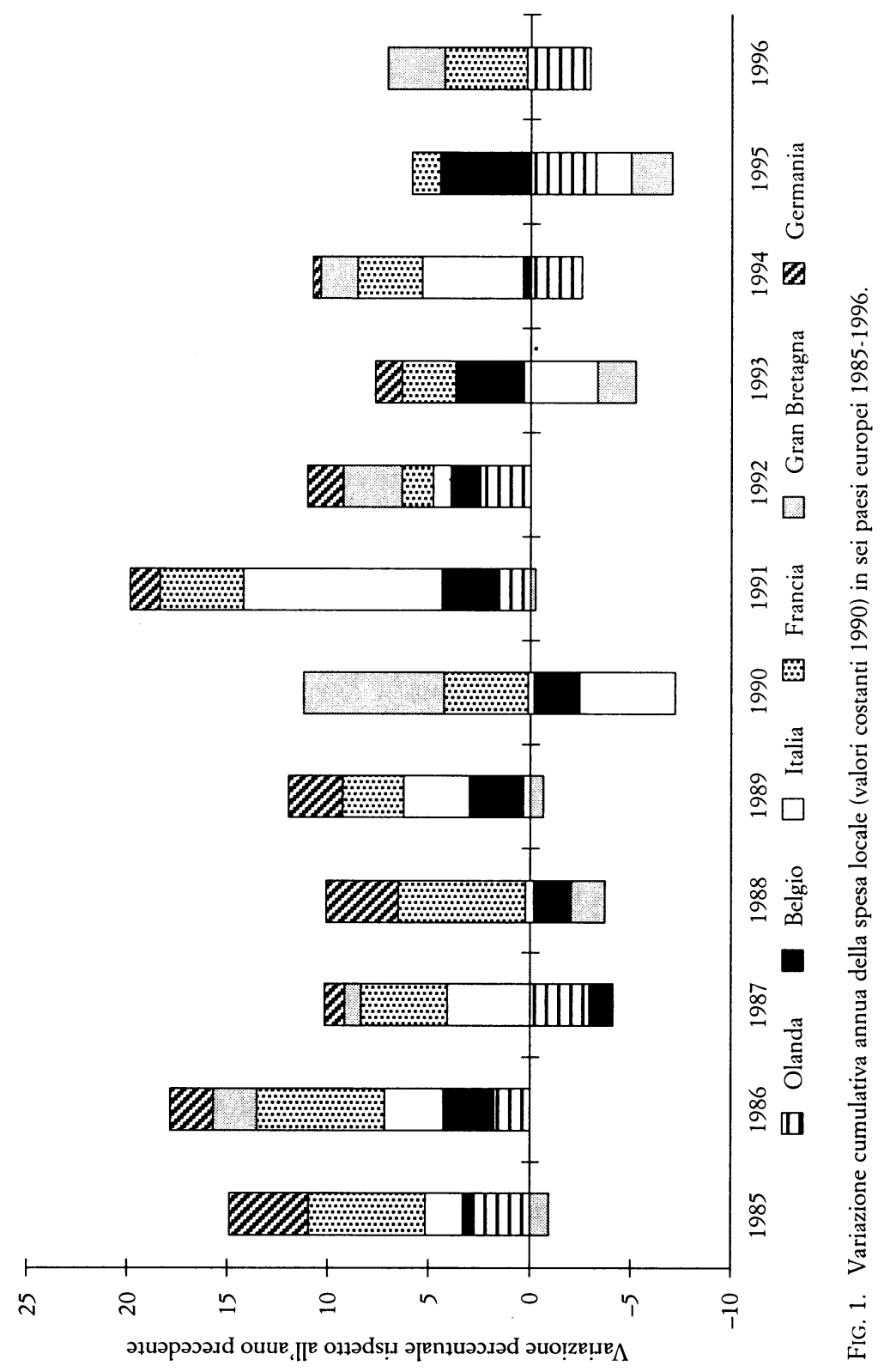


l'Olanda, l'Italia e la Francia. Aggiungerò comunque alcune considerazioni anche sulla Spagna, che ha visto esattamente in questo periodo una significativa modificazione dell'equilibrio centro-periferia.

Come scaricare la patata bollente dal centro alla periferia: $i$ casi dell'Olanda e dell'Italia

Cominciare dai paesi tradizionalmente posizionati all'estremità opposta rispetto a quelli scandinavi, almeno per quanto riguarda i rapporti intergovernativi, può risultare particolarmente istruttivo. Tornando per un istante ai dati contenuti nelle tabelle 1 e 2, troviamo un preciso riscontro di come l'Olanda e l'Italia (seguite a ruota dalla Francia) fossero i paesi con il più alto grado di centralizzazione politico-amministrativa e il minor grado di autonomia fiscale a livello locale. Ebbene, se estendiamo l'orizzonte agli anni ottanta e novanta, ciò che colpisce immediatamente è l'estrema somiglianza del percorso seguito in entrambi i paesi per quanto riguarda l'evoluzione dei rapporti centro-periferia e le conseguenti ripercussioni sul livello locale.

Come si evince dalla figura 2, a metà degli anni ottanta, tanto in Olanda quanto in Italia un terzo circa della spesa pubblica corrente era assorbito dai livelli sub-nazionali di governo ${ }^{10}$. Tuttavia, dieci anni più tardi in entrambi i paesi tale quota si era ridotta a poco più di un quarto del totale. Per l'esattezza questa comune flessione, riflette traiettorie tra loro distinte. Nel caso olandese (cfr. fig. 1) possiamo infatti registrare nell'arco di tutto il periodo considerato una situazione di blocco o di contrazione nei livelli di spesa locale (sempre in valori costanti). Evidentemente il passaggio dalla «sindrome» al «miracolo» non è stato senza conseguenze per gli enti locali olandesi! In Italia invece gli anni ottanta segnano ancora un periodo di crescita della spesa locale in buona parte attribuibile alla espansione della componente sanitaria. Il trend di crescita si interrompe solo all'inizio degli anni novanta.

10 Mentre in Olanda l'incidenza della spesa locale era già molto consistente nei decenni precedenti, in Italia la crescita della quota di spesa pubblica erogata a livello sub-nazionale è dovuta soprattutto al passaggio della spesa sanitaria dal sistema mutualistico al sistema sanitario nazionale, con il conseguente conferimento delle responsabilità di spesa ai governi regionali e alle unità sanitarie locali. 







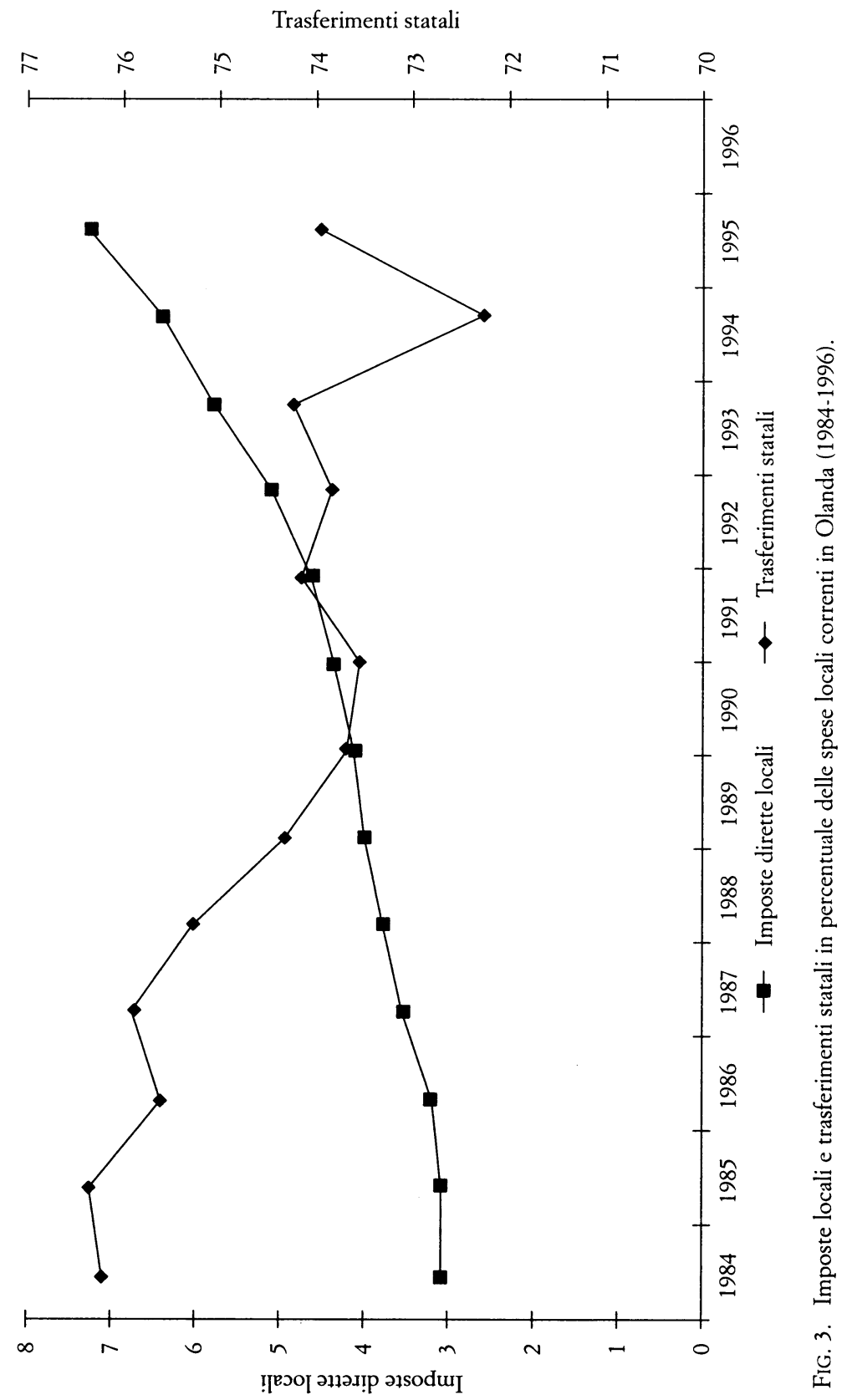


Trasferimenti statali

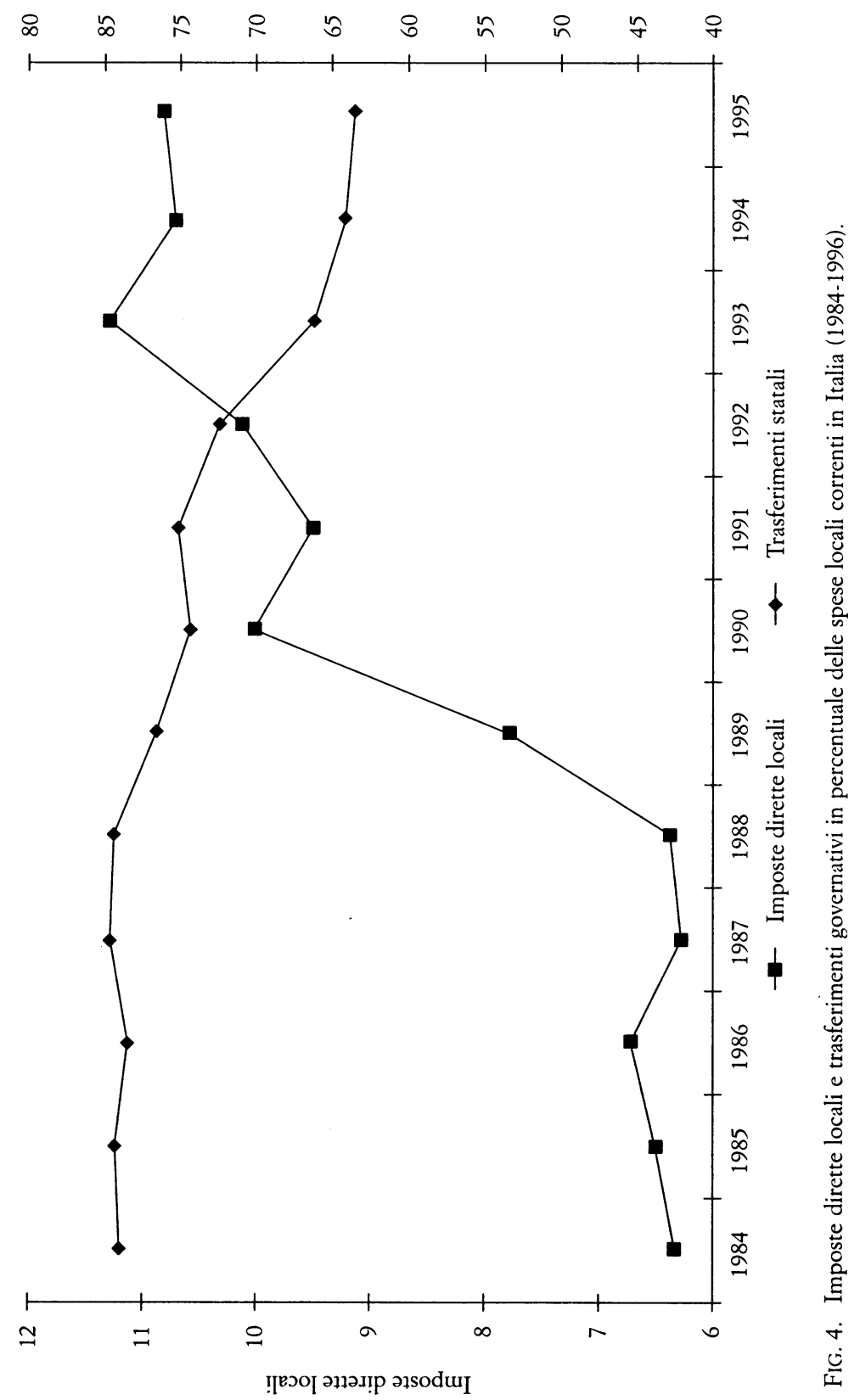


$\mathrm{Ma}$ forse è ancor più interessante mettere in luce quanto è avvenuto in questo frattempo sul versante delle entrate. Come si può ricavare dalle figure 3 e 4, l'autonomia impositiva locale, pur avendo ancora un'incidenza contenuta come fonte di copertura delle spese locali, è stata per la prima volta significativamente ampliata, toccando livelli che non aveva mai raggiunto in precedenza; una scelta chiaramente destinata a compensare la riduzione dei trasferimenti da parte del governo centrale.

Olanda. Nel caso dell'Olanda, durante gli ultimi cinquant'anni le imposte locali non sono mai state in grado di coprire più di un esiguo $3 \%$ della spesa corrente locale. Tutt'a un tratto invece questa fonte di entrata ha raddoppiato il suo peso. Se combiniamo questo dato con le informazioni fornite poco sopra in ordine al calo della spesa locale, è evidente come le prospettive per il settore di policy di cui ci stiamo occupando appaiano assai poco rosee. Non possiamo ad esempio trascurare che un aumento dell'autonomia impositiva locale - proprio mentre erano in corso pesanti manovre di risanamento della finanza pubblica - difficilmente avrebbe potuto trovare un'accoglienza entusiastica da parte di enti locali totalmente disabituati a considerare i propri cittadini come contribuenti; a maggior ragione in un contesto in cui ulteriori aumenti della pressione fiscale rappresentavano in sostanza un'opzione politicamente non perseguibile.

Queste considerazioni risultano decisamente pertinenti alla luce delle nuove esigenze che andavano delineandosi in Olanda per quanto riguarda le funzioni di cura dell'infanzia in particolare, soprattutto a partire dagli anni ottanta. Fino al decennio precedente, il tasso di occupazione femminile olandese era il più basso di tutta l'Europa continentale, ma nell'arco degli anni ottanta la situazione ha subito un'improvvisa accelerazione, al punto che nel giro di pochi anni il paese ha raggiunto i livelli medi europei. Anche se la maggioranza delle donne ha assunto lavori part-time, il nuovo scenario ha sicuramente contribuito a mettere in discussione la percezione tradizionale del ruolo della donna, per quanto riguarda in particolare l'accudimento della prima infanzia. Jet Bussemaker (1998) ci fornisce in proposito una stimolante ricostruzione di come la retorica politica e gli assunti ideologici che avevano fino a quel momento ostacolato qualsiasi significativo coinvolgimento dello stato nella realizzazione di servizi per l'infanzia avesse ceduto il posto negli anni 
ottanta ad una nuova impostazione favorevole all'assunzione di un impegno pubblico diretto.

$\grave{E}$ in questo contesto che per la prima volta il governo olandese ha varato un provvedimento legislativo teso ad espandere $\mathrm{i}$ servizi per la prima infanzia. Ma a questo punto qualsiasi soluzione di tipo scandinavo era chiaramente esclusa dal ventaglio delle alternative possibili. Peraltro, a partire dal 1996, le competenze in questo settore sono state trasferite dal livello nazionale a quello locale. Ma alla luce di quanto abbiamo detto fin qui, non possiamo non aspettarci dal coinvolgimento degli enti locali implicazioni ed effetti profondamente diversi rispetto a quelli derivanti dalla analoga strategia seguita dai paesi scandinavi venticinque anni prima. Gli enti locali olandesi si sono infatti trovati - per così dire - «schiacciati» tra una crescente domanda di servizi e la difficoltà sia politica che economica di aumentare la pressione fiscale per potervi far fronte.

Questa posizione - a dir poco - scomoda non è certo confinata ai servizi per la prima infanzia. Come è noto, in Olanda la segmentazione sub-culturale ha dato vita ad un modello di solidarietà imperniato su pilastri politico-religiosi nettamente distinti (Ferrera 1993, 165-174; Van Kersbergen 1995), nel cui ambito si è realizzata una proliferazione estrema delle organizzazioni di volontariato dotate però di strutture di vertice nazionali, la cui forza ha portato addirittura a parlare di «quinto potere» (Kramer, Lorentzen, Melief e Pasquinelli 1993). Come documentato dagli studi sull'argomento, tali strutture hanno «cortocircuitato» le amministrazioni locali, rivolgendosi direttamente al centro per ottenere finanziamenti e riuscendo ad acquisire una posizione quasi monopolistica nella erogazione dei servizi sociali. La situazione è cambiata ben poco anche a seguito delle misure di decentramento avviate in questi ultimi anni dal livello centrale. Esattamente come nel caso degli asili nido, il governo nazionale - per alleggerire la pressione nei suoi confronti - ha cercato di scaricare «la patata bollente» sui governi locali. Ma dato il quadro dei vincoli in cui gli enti locali hanno dovuto operare negli anni novanta, questi ultimi si sono trovati in sostanza a fare da filtro nei confronti del terzo settore o - a seconda dei casi - nei confronti di datori di lavoro, associazioni di cittadini, e fornitori privati di servizi.

Italia. Se volgiamo a questo punto l'attenzione al caso italiano, nonostante la diversità degli elementi specifici, le dinamiche 
in atto appaiono largamente simili e - se possibile - ancor più accentuate. Tutte e tre le principali tendenze, che abbiamo enucleato analizzando il caso olandese, sono presenti anche in quello italiano. Innanzitutto, la legislazione nazionale ha ampliato sia le competenze regionali che locali; in secondo luogo, la quota di spesa pubblica assorbita dai livelli sub-nazionali è diminuita; ed infine l'autonomia impositiva locale è significativamente aumentata, soprattutto a partire dagli anni novanta. Per inquadrare correttamente i recenti sviluppi delle politiche socio-assistenziali nel nostro paese occorre comunque prendere in considerazione anche il ruolo storicamente esercitato dalle istituzioni caritative e l'incidenza della divaricazione territoriale tra Nord e Sud.

Grazie alla posizione privilegiata della Chiesa e alla forte tradizione cattolica del paese, l'Italia ha storicamente avuto un numero assai più elevato di enti caritativi di matrice religiosa rispetto agli altri paesi europei. In particolare, le istituzioni pubbliche di beneficenza e assistenza (le cosiddette Ipab) hanno potuto operare per un secolo nella massima autonomia ed indipendenza, agendo in pratica come soggetti privati, nonostante la loro natura formalmente pubblica ${ }^{11}$. Insieme agli istituti religiosi, le Ipab hanno sempre gestito la stragrande maggioranza delle strutture per anziani, per minori e per handicappati, in un contesto caratterizzato dall'assoluta marginalità delle politiche socio-assistenziali, da una estrema frammentarietà degli interventi e da un ruolo del tutto secondario degli enti locali.

L'istituzione delle regioni nel 1970 ha offerto una significativa opportunità di modernizzazione per il settore, ma i risultati sono stati tutt'altro che omogenei all'interno del paese. L'altro aspetto da tenere presente attiene appunto alla dimensione ter-

11 Può essere forse di un qualche interesse ricordare che le opere pie hanno originariamente acquisito una veste pubblica in base alla legge Crispi del 1890. Quasi un secolo più tardi la Corte costituzionale ha imposto una inversione di rotta. La sentenza della Corte n. 396 del 1988 ha infatti stabilito l'illegittimità dell'articolo 1 della legge del 1890 per contrasto con l'articolo 38 della Costituzione. In conseguenza, dopo aver gravitato per oltre cento anni nella sfera pubblica, le opere pie che soddisfano determinati requisiti possono essere oggi privatizzate. Merita aggiungere che ancora nel 1977, il governo aveva varato un provvedimento (il D.P.R. 616) che prevedeva l'estinzione di tutte le Ipab ed il passaggio di beni, risorse e personale alle regioni e da queste ai comuni. A distanza di pochissimi anni le regioni si sono così trovate a dar seguito a politiche di segno diametralmente opposto e sono oggi impegnate nella regolamentazione del processo di privatizzazione anziché nel sostanziale annullamento delle opere pie a favore del potere locale. 
ritoriale. Se guardiamo infatti all'invecchiamento della popolazione, all'occupazione femminile e ai cambiamenti della struttura familiare, le regioni del Centro-Nord appaiono sostanzialmente allineate con il resto dell'Europa continentale e dell'Europa del Nord. Per converso, gli indicatori sociali relativi al Mezzogiorno risultano assai più simili a quelli della Grecia, del Portogallo e di alcune regioni della Spagna dove il problema cruciale per un gran numero di famiglie è l'elevato tasso di dipendenza e l'insufficienza dei redditi da lavoro, se non addirittura la loro mancanza totale. Le due parti del paese insomma differiscono sia per quanto riguarda la natura dei bisogni sociali sia per quanto riguarda la percezione culturale di tali bisogni. $\mathrm{Ma}$ occorre subito aggiungere che le politiche messe in atto dai governi regionali, lungi dal colmare il divario tra le due parti del paese, hanno ulteriormente contribuito ad accentuarlo.

Come noto, la Costituzione italiana ha attribuito alle regioni poteri legislativi nel settore dell'assistenza pubblica e dei servizi sociali; ma il Parlamento non ha mai provveduto ad approvare la relativa legge-quadro, consentendo di fatto ai governi regionali di differenziare le rispettive politiche in misura assai più consistente di quanto istituzionalmente previsto. Mentre dunque le regioni meridionali hanno in larga misura mantenuto intatto l'arcaico sistema di assistenza pubblica ereditato dallo stato, le regioni del Nord e del Centro si sono impegnate fin dall'inizio sul fronte dell'innovazione, promuovendo la realizzazione di un'ampia gamma di servizi territoriali (Fargion 1997b). In particolare, le regioni guidate da maggioranze di sinistra hanno puntato alla costruzione di una rete capillare di servizi sociali direttamente gestiti dagli enti locali.

Dati i meccanismi di finanziamento delle regioni, queste ultime tuttavia erano in grado di fornire solo una copertura finanziaria molto limitata per l'attivazione dei nuovi servizi, ed hanno rimesso sostanzialmente il compito agli enti locali, i quali a loro volta non erano certo in condizioni migliori per dar corso alle nuove previsioni legislative. I comuni dipendevano infatti pressoché esclusivamente dai trasferimenti statali e, soprattutto dagli anni ottanta in poi, hanno dovuto misurarsi con politiche restrittive attuate dal centro ed orientate da un lato a contenere il finanziamento degli enti locali e dall'altro a imporre il blocco delle assunzioni locali. In breve, $\mathrm{i}$ comuni del Centro-Nord erano ancora impegnati nella fase iniziale di attuazione della legislazione regionale quando hanno dovuto fare $\mathrm{i}$ conti con tutta 
una serie di restrizioni imposte dal centro. E va detto che questo approccio del livello nazionale verso i poteri locali risulta in stridente contrasto con le assai poco oculate pratiche di spesa prevalenti in quasi tutti gli altri settori. Quel che è certo è che, nonostante gli sperperi del centro, il governo locale in Italia ha in buona parte condiviso quel clima di austerità e di ristrettezze largamente prevalente nel resto d'Europa. Proprio come suggerivamo poco sopra per l'Olanda, gli enti locali - soprattutto nelle aree urbane - si sono trovati sempre più «schiacciati» tra una pressione crescente a favore della istituzione di nuovi servi$\mathrm{zi}$, peraltro pienamente legittimati dalla legislazione regionale, e l'impossibilità di provvedervi direttamente, soprattutto in conseguenza del blocco delle assunzioni di personale imposto dal centro.

Date queste coordinate, è più agevole comprendere come $\mathrm{e}$ perché gli enti locali si siano rivolti con sempre maggior frequenza ed intensità ad un variegato arco di soggetti non pubblici operanti soprattutto nell'ambito del terzo settore ${ }^{12}$. Questi ultimi consentivano infatti di fornire una risposta di policy, per quanto limitata, aggirando il blocco delle assunzioni. Spesso sono stati gli stessi comuni a stimolare la creazione di nuove organizzazioni non profit, soprattutto cooperative sociali, dal momento che i vecchi enti di settore in molti casi non apparivano in grado di rispondere alle nuove domande. Le cooperative potevano facilmente provvedere alla fornitura di una vasta gamma di servizi, inclusa l'assistenza domiciliare, offrendo al tempo stesso uno sbocco al problema della disoccupazione giovanile, che andava assumendo dimensioni sempre più preoccupanti.

Il risultato è stato un complesso intreccio di rapporti pubblico-privato anche in quelle regioni, come la Toscana e l'Emilia-Romagna, che avevano inizialmente cercato di dar vita ad una versione italiana del modello scandinavo, imperniata sulla centralità del comune e dei servizi pubblici. Come si può ben vedere, il timing appare cruciale per un corretto inquadramento delle politiche socio-assistenziali nel caso italiano. La serie di eventi da cui è scaturita l'istituzione delle regioni ha offerto - se vogliamo - una opportunità inattesa per affrontare le nuove domande connesse al processo di «defamilizzazione», ma le condi-

12 Per un'analisi del ruolo cruciale che le famiglie hanno sempre svolto nell'accudimento di bambini, anziani e portatori di handicap si veda in particolare Trifiletti (1998). 
zioni avverse della particolare congiuntura storica hanno reso ben presto impraticabile il modello scandinavo, a prescindere dalle maggioranze politiche di governo.

Ma quali sono i tratti salienti del sistema che si è andato consolidando in questi ultimi due decenni? Come suggerisce lucidamente Ranci (1999), volendo cogliere la natura dei rapporti tra enti locali e terzo settore è meglio parlare di «mutuo aggiustamento» che non di privatizzazione. Secondo l'autore, le élites politiche locali hanno teso prevalentemente a sviluppare rapporti particolaristici e legami di tipo clientelare con il terzo settore, con l'ovvio risultato di rafforzare una serie di mercati protetti anziché stimolare una aperta competizione tra i diversi fornitori di servizi. Tuttavia, così come per la vita politica del paese e per l'amministrazione dello stato, gli anni novanta sembrano rappresentare un punto di svolta anche in questo campo. In contrasto con il sostanziale disinteresse dimostrato a livello nazionale per le politiche socio-assistenziali durante tutti gli anni ottanta, il decennio successivo si apre ad esempio con l'approvazione da parte del Parlamento delle leggi-quadro sul volontariato e sulle cooperative sociali, cui fa seguito negli anni successivi una regolamentazione delle delicate questioni relative al regime fiscale delle organizzazioni senza scopo di lucro (Ascoli 1999).

Sebbene nelle regioni del Sud si registri ancora un forte ritardo, le nuove disposizioni legislative hanno sicuramente stimolato gli enti locali a ridefinire i loro rapporti con il terzo settore. Soprattutto nel Centro-Nord molti comuni si erano già mossi in questa direzione, ma il processo è ancora ovunque in itinere. Le questioni sul piatto della bilancia sono davvero molte e vanno - per citarne solo alcune - dalla definizione degli standard quantitativi e qualitativi dei servizi, alle procedure di monitoraggio e valutazione dei risultati e più in generale all'ampiezza della competizione tra fornitori. Qualunque sia il risultato di queste sfide, resta il fatto che fino ad oggi il terzo settore è stato pressoché totalmente dipendente dal finanziamento degli enti locali. Ma i margini di manovra da parte di questo livello di governo appaiono oggi estremamente limitati. Come indica la figura 4 , i trasferimenti statali hanno subito una significativa riduzione. L'aumento dell'autonomia impositiva avrebbe dovuto controbilanciare tale caduta, ma almeno fin qui non si è previsto alcun meccanismo compensativo tra la pressione fiscale nazionale e quella locale. L'imposizione locale è risultata così me- 
ramente aggiuntiva rispetto a quella nazionale ed in un contesto caratterizzato da una elevata pressione fiscale complessiva diventa estremamente difficile per gli enti locali ipotizzare una espansione delle loro responsabilità finanziarie. In questa particolare congiuntura storica, l'attribuzione di una maggior autonomia impositiva non è detto che sia poi un dono così gradito!

Decentramento finanziariamente sostenuto dal centro: $i$ casi della Francia e della Spagna

La Francia e la Spagna riflettono una versione diversa del processo di decentramento che ha investito l'Europa tra gli anni ottanta e novanta. In questi due paesi i rapporti tra centro e periferia non si configurano nei termini di un gioco a somma zero in cui il livello locale è destinato ad un ruolo perdente. Al contrario, come documentato dalla figura 5, alla fine del periodo da noi considerato gli enti sub-nazionali controllavano una quota delle risorse pubbliche complessive decisamente più rilevante.

Del resto è anche piuttosto facile identificare i provvedimenti che hanno provocato questo aumento. Nel caso della Francia le riforme del periodo 1982-86 hanno attribuito ai dipartimenti la gran parte delle competenze nel settore dell'assistenza pubblica, facendo lievitare notevolmente i loro bilanci (fino a quel momento assolutamente irrisori). A seguito dell'introduzione nel 1989 del reddito minimo di inserimento (il cosiddetto Rmi) i dipartimenti hanno inoltre assunto a loro carico il finanziamento del $20 \%$ del costo del programma, destinato a coprire le attività di integrazione sociale. Nel caso della Spagna l'aumento più significativo della spesa locale e regionale si è verificato nella seconda metà degli anni ottanta in connessione all'avvio concreto della riforma sanitaria, che ha attribuito ai governi regionali la responsabilità del settore. Il coinvolgimento delle regioni nella gestione del reddito minimo e degli altri programmi di assistenza pubblica ha fornito un contributo ulteriore, anche se più limitato. L'avvio di un programma di reddito minimo particolarmente generoso da parte dei paesi baschi, che godono di condizioni privilegiate dal punto di vista dell'autonomia fiscale, ha infatti innescato una sorta di domino effect (Moreno e Arriba 1999) che ha portato ad una competizione al rialzo tra le regioni. 





Ma come è che gli enti locali sono riusciti a rafforzare il loro ruolo e soprattutto ad ampliare la fetta di risorse da essi controllata a discapito di altri soggetti pubblici? La risposta risiede, almeno in parte, nella diversa strategia di finanziamento seguita in questi paesi rispetto all'Olanda, all'Italia e - se per questo - anche al Belgio. Come illustrano le figure 6 e 7 non siamo di fronte ad una caduta nel livello dei trasferimenti statali, il che significa che il governo locale tanto in Francia quanto in Spagna ha avuto meno vincoli finanziari rispetto ai paesi limitrofi. Ma nonostante questa posizione di vantaggio e nonostante la particolare enfasi posta in entrambi i paesi sulla nuova articolazione locale dei poteri, l'evoluzione delle misure adottate nel settore dei servizi sociali non sembra averne tratto particolare beneficio. Se questo vale per entrambi i paesi, le ragioni a monte appaiono in realtà sostanzialmente diverse nei due casi. Partiamo dalla Francia.

Non vi è dubbio che durante gli anni ottanta e ancor più negli anni novanta, la Francia ha visto crescere significativamente la domanda di servizi per l'infanzia e per gli anziani non-autosufficienti, ma a quel punto l'arena di policy era ormai fortemente strutturata e densamente organizzata, il che limitava di fatto in termini stringenti le alternative di policy. Per cogliere a pieno le implicazioni di questo stato di cose, conviene richiamare velocemente alcuni aspetti che in questo periodo hanno caratterizzato l'evoluzione complessiva del welfare state francese. Come noto, in questo dopoguerra la Francia ha sviluppato un sistema di protezione di tipo occupazionale, essenzialmente imperniato sulla tutela assicurativa della forza lavoro attiva. In tale contesto gli enti assicurativi, che rappresentavano l'asse portante del sistema, hanno provveduto ad erogare un ventaglio estremamente frammentato e diversificato di prestazioni e servizi alle varie categorie occupazionali. Fino agli anni ottanta la questione dei servizi di assistenza è stata affrontata entro questo quadro di riferimento. Più precisamente, sia la rete delle Caisses d'allocations familiales (Caf) sia la Caisse nationale d'assurance vieillesse des travailleurs salariés (Cnavts) sono attivamente intervenute per sostenere da un punto di vista finanziario la realizzazione rispettivamente di servizi per la prima infanzia e di assistenza domiciliare per gli anziani, anche se ovviamente questi interventi sono rimasti del tutto secondari rispetto alla gestione dei programmi di garanzia del reddito.

Nei primi anni ottanta, durante la presidenza Mitterrand, a 


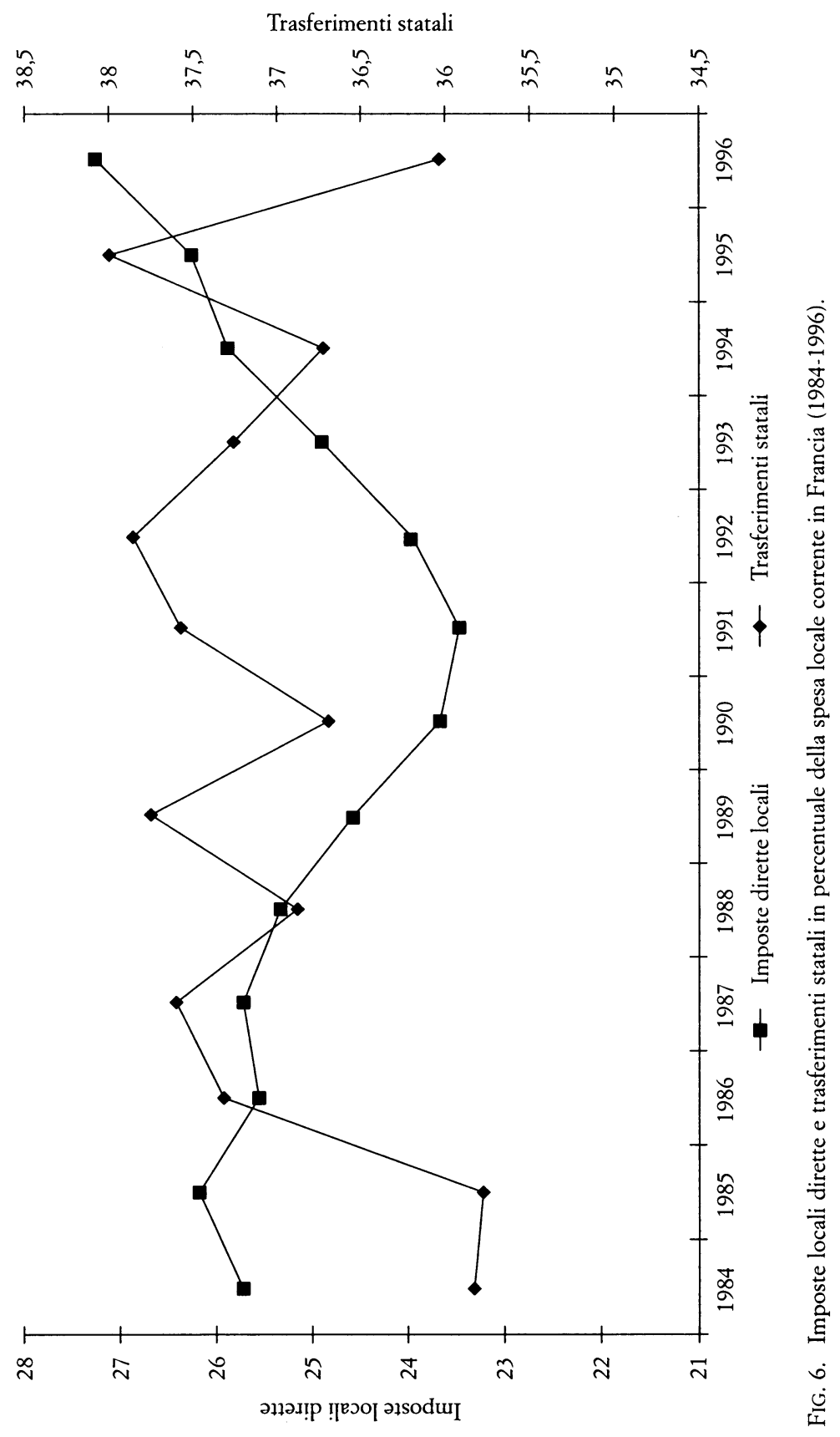


Trasferimenti statali




tale sistema di protezione si è aggiunta una seconda componente non contributiva, nota come solidarieté nationale, destinata a garantire una rete di protezione di base nei confronti delle persone in condizioni di povertà ed esclusione sociale. La crescita estremamente sostenuta di questa nuova componente del welfare state francese, oltre a rappresentare il cambiamento più significativo degli ultimi due decenni, ha prodotto un marcato dualismo nel sistema di protezione sociale di questo paese. Queste «nuove politiche sociali» sono appunto quelle che hanno costituito il principale oggetto del processo di decentramento del periodo 1982-1986 cui accennavamo più sopra. Nella sua puntuale ricostruzione di tali sviluppi, Palier (1998) sottolinea appunto il forte contrasto tra la dimensione territoriale del nuovo approccio e la logica categoriale cui è improntato tutto il sistema assicurativo. Ai nostri fini è comunque ancor più importante sottolineare che le misure di decentramento attuate nei confronti dei comuni e dei dipartimenti, nel momento in cui provvedevano all'attribuzione di nuovi compiti, hanno anche incanalato l'azione locale su di una traiettoria ben definita. Più precisamente, gli enti locali sono stati chiamati a fornire una articolata gamma di prestazioni e servizi esclusivamente destinati alla fetta più povera della popolazione ed accessibili solo sulla base di una rigorosa prova dei mezzi. Questo ha chiaramente precluso la possibilità da parte degli enti locali di realizzare una rete di servizi collettivi destinati alla generalità della popolazione. Gli enti locali hanno finito così per rappresentare solo l'ultimo dei tanti soggetti operanti in un settore estremamente frammentato e diversificato. Come suggerisce uno studio comparato dell'Ocse sull'assistenza agli anziani non-autosufficienti:

in Francia la molteplicità delle fonti di finanziamento dell'assistenza impone agli anziani e alle loro famiglie di negoziare con un gran numero di soggetti istituzionali per mettere insieme «un pacchetto» di interventi [...] la mera complessità delle procedure di copertura dei costi per l'assistenza domiciliare unita all'incertezza in ordine alla possibilità di riuscire a mettere insieme tutte le componenti del «pacchetto» costituisce un elemento di freno rispetto alla alternativa domiciliare quando occorre affrontare un problema di assistenza $(1996,135)$.

Per completare il quadro occorre aggiungere che le dinamiche in esame interagiscono con una sequenza causale del tutto indipendente, la quale ha a che fare con l'aggravarsi del problema della disoccupazione. Claude Martin (1999) richiama giusta- 
mente l'attenzione su questo aspetto nel fornire una chiave di lettura dei recenti sviluppi in quest'area di policy. In effetti, nel dibattito politico e sociale i servizi territoriali (services de proximité) vengono costantemente abbinati ad una immagine particolarmente eloquente che è quella di gisement d'emploi. Non a caso i recenti provvedimenti volti a conciliare la vita professionale con quella familiare puntano a creare il più vasto arco possibile di opportunità di lavoro. Per l'assistenza alla prima infanzia questo ha voluto dire soprattutto favorire (attraverso tutta una serie di incentivi fiscali) soluzioni di tipo individuale tese a garantire la cura dei bambini entro le mura domestiche da parte di apposite figure professionali. Ma se estendiamo lo sguardo all'intero arco dei servizi territoriali, ci troviamo di fronte ad una proliferazione di organizzazioni senza scopo di lucro ed in particolare di associazioni di volontariato, che nel quadro del nuovo welfare mix appaiono come i soggetti più in grado di fornire risposte flessibili e a basso costo (Laville 1996). Per la verità, anche guardando al futuro, appare piuttosto improbabile che i comuni assumano un ruolo più attivo, vista la estrema frammentazione territoriale e la grande variabilità delle risorse connessa al peso che rivestono le imposte locali sulle attività produttive.

La Spagna offre un panorama non meno preoccupante, anche se del tutto diverso. Gli studi disponibili descrivono unanimemente il livello dei servizi sociali pubblici come assolutamente minimale e non sembrano esservi segnali di alcun possibile miglioramento, almeno nell'immediato futuro. Ed è certo che non possiamo imputare questo stato di cose alla debolezza del potere locale e regionale; in questi ultimi anni l'equilibrio dei rapporti centro-periferia si è infatti spostato sempre più a favore dei livelli sub-nazionali. In questo caso sono soprattutto fattori di ordine culturale che hanno ostacolato un preciso impegno pubblico su questo fronte. Ripercorrendo i sondaggi di opinione disponibili, Chulià e Alvarez-Miranda (1999) sottolineano come quattro adulti su cinque ritengono che, in caso di necessità di assistenza da parte dell'anziano, spetti ai figli o comunque ai familiari provvedere. Ma tutti gli indicatori sociali suggeriscono che la società sta cambiando davvero molto rapidamente in Spagna ${ }^{13}$ ed il paese potrebbe trovarsi presto a do-

13 Oggi ad esempio l'assistenza agli anziani è quasi esclusivamente fornita da donne con livelli di istruzione estremamente bassi, ma il rapido aumento dei livelli di scola- 
ver affrontare una situazione di vera e propria crisi per quanto riguarda l'assistenza alle fasce più deboli senza avere la benché minima preparazione.

\section{Tornando ai paesi scandinavi}

Tornando ai paesi scandinavi da cui aveva preso le mosse il nostro discorso, non vi è dubbio che anche essi abbiano dovuto fare $\mathrm{i}$ conti con le avverse condizioni economiche e finanziarie, che hanno investito il resto dell'Europa nell'arco degli ultimi due decenni. Né può meravigliare che ciò abbia avuto ripercussioni negative anche sul governo locale, visto che quest'ultimo «faceva la parte del leone» nella ripartizione della spesa pubblica (Blom-Hansen 1998). A questo proposito la figura 8 non lascia adito a dubbi. Eccetto la Danimarca, che ha in larga misura recuperato il crollo nei livelli di spesa locale verificatosi a metà degli anni ottanta, negli altri tre paesi scandinavi la percentuale di spesa locale rispetto alla spesa pubblica complessiva era decisamente più bassa nel 1996 rispetto a dieci anni prima, con il calo più vistoso in Svezia ${ }^{14}$. Ciò nondimeno, in Scandinavia la quota di risorse correnti controllata dal potere locale è rimasta notevolmente più consistente rispetto al resto dell'Europa.

Se spostiamo l'attenzione sul versante delle entrate, anche qui troviamo un aumento nella incidenza delle imposte locali dirette - come fonte di finanziamento della spesa locale - analogo a quello riscontrato per altri paesi europei. Le informazioni contenute in proposito nella figura 9 sono estremamente esplicite. La Norvegia è l'unico paese che non sembra risentire della tendenza generale all'aumento nel ruolo della tassazione diretta locale; ma non dobbiamo dimenticare che le finanze pubbliche in questo paese godono di condizioni del tutto privilegiate grazie alle risorse energetiche recentemente scoperte nei mari del Nord.

rizzazione che ha segnato gli ultimi anni porterà presto a far quasi scomparire questo particolare profilo femminile.

14 Come noto, gran parte della spesa locale è costituita da consumi pubblici finali. A questo proposito nel discutere le recenti misure restrittive che a loro avviso conterrebbero una precisa pregiudiziale negativa nei confronti dei servizi (anti-service bias), Clayton e Pontusson sostengono correttamente: «la spesa per consumi pubblici finali ha continuato a crescere in termini reali durante tutti gli anni ottanta, ma è cresciuta meno rapidamente rispetto ai trasferimenti sociali» $(1998,95)$. 


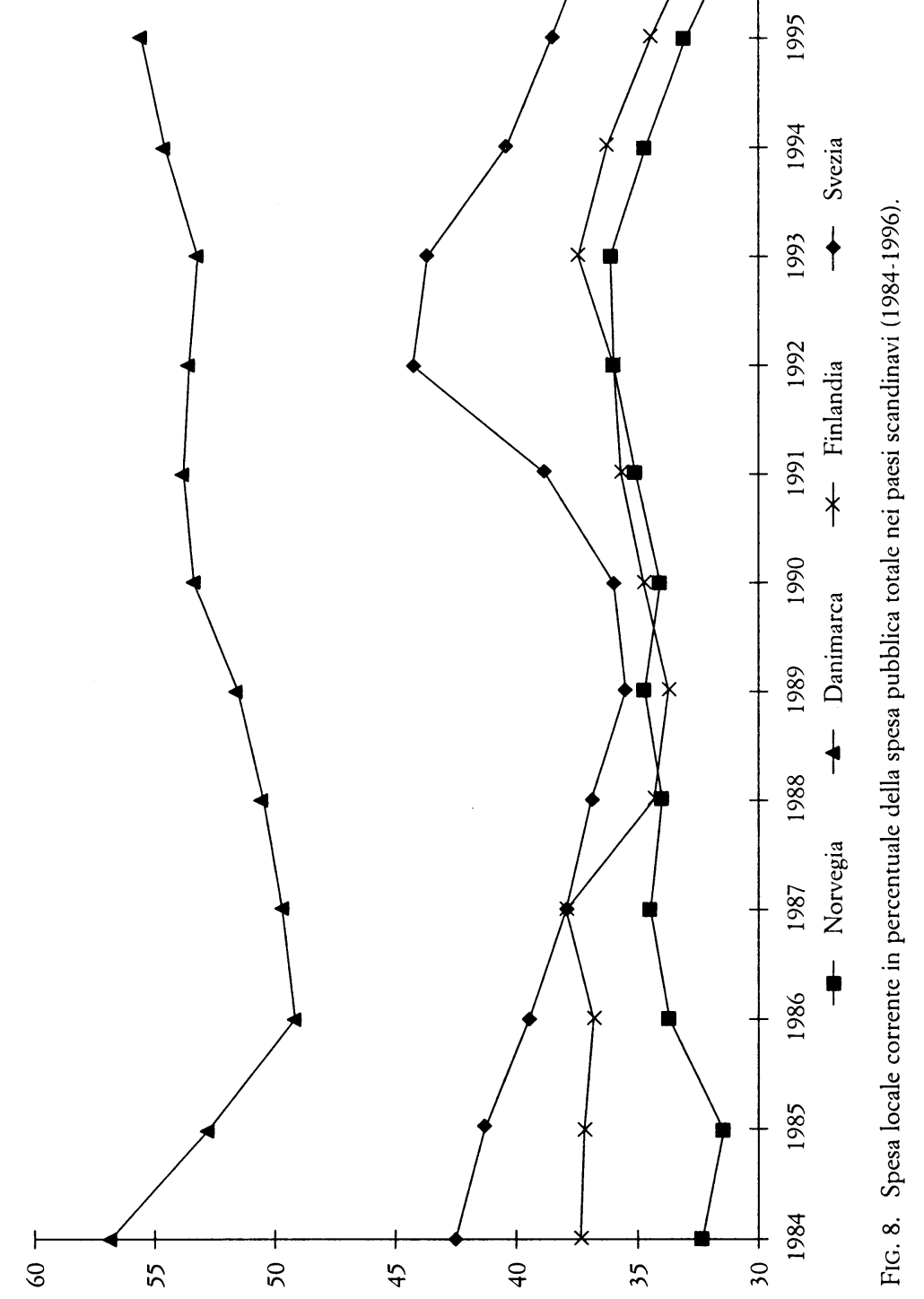




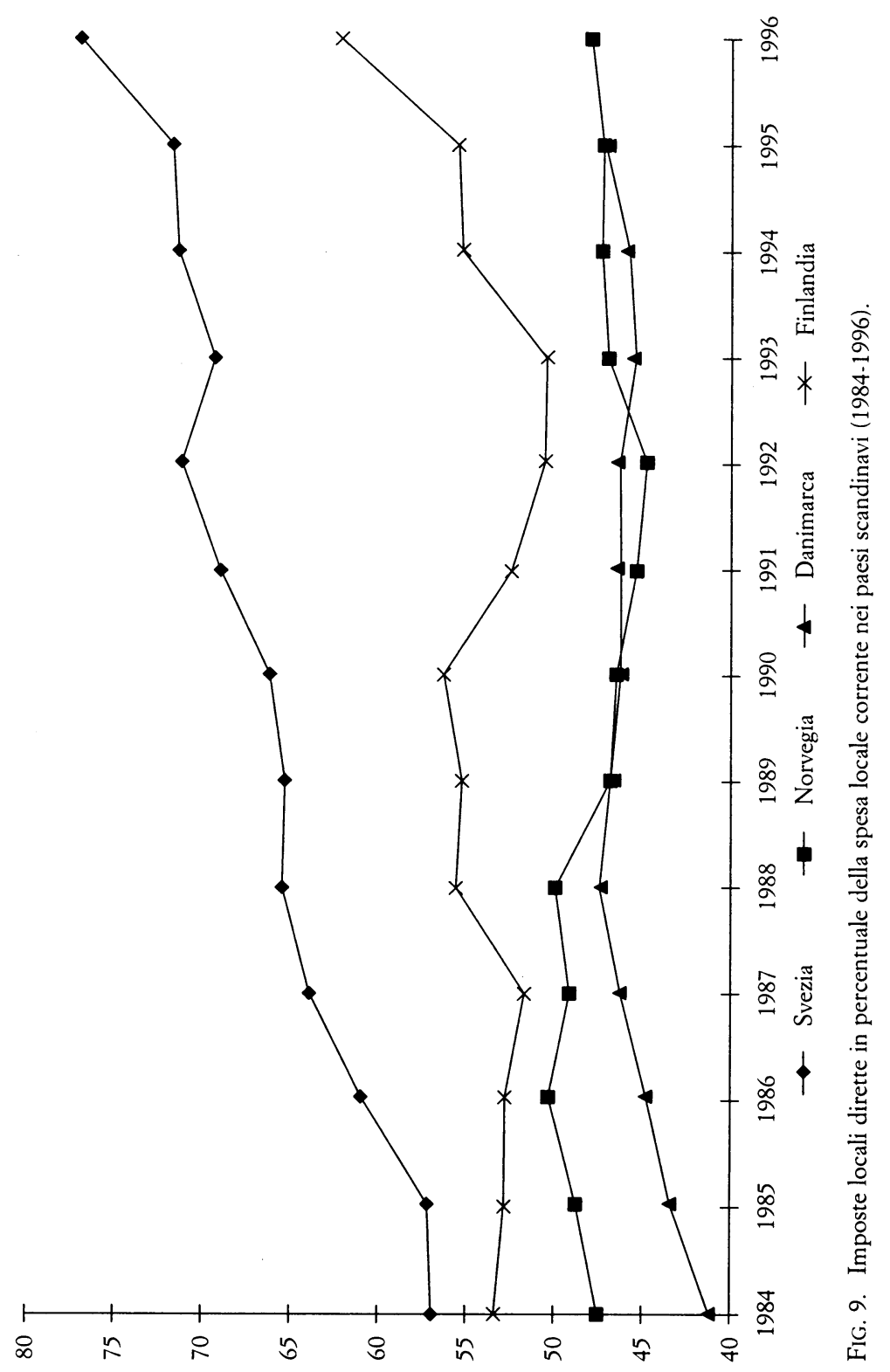


Sebbene le imposte locali coprissero già una quota consistente della spesa locale, questa strategia è stata ulteriormente rafforzata, al punto che oggi in Svezia ben due terzi dei bilanci locali sono finanziati attraverso la tassazione locale! Tutto questo fa parte di una linea politica tesa ad ampliare i poteri discrezionali a livello locale, come emblematicamente testimoniato dalla sperimentazione norvegese su quelli che vengono chiamati «comuni liberi»; una linea che implica in primo luogo un allentamento rispetto alla precedente rigida definizione da parte del livello nazionale di criteri e parametri uniformi per tutta una serie di servizi, ivi inclusi in particolare quelli sociali.

Fermo restando tutto questo, gli studi disponibili documentano come il grosso dei servizi sociali sia ancora fornito dal settore pubblico e come $\mathrm{i}$ livelli di copertura restino incomparabilmente più alti rispetto a qualsiasi altro paese. Nel caso della Svezia, in particolare, da più parti si sottolinea come «l'interesse politico per il volontariato è rimasto in larga misura a livello retorico» (Jeppsson Grassman 1998) ${ }^{15}$. Con questo non voglio assolutamente sostenere che il modello dei servizi sociali scandinavo sia rimasto immune da processi di riorganizzazione. In uno studio sulla articolazione attuale dei servizi sociali in sette paesi europei, particolarmente ricco di informazioni, Rostgaard e Fridberg (1999) documentano lo snellimento del sistema dei servizi, la eliminazione di procedure di erogazione open-ended e la concentrazione delle risorse sui casi a più elevato bisogno assistenziale; ma illustrano anche lo scarto che permane negli altri paesi europei da essi considerati, specialmente per quanto riguarda l'assistenza domiciliare ed i servizi territoriali in generale ${ }^{16}$.

Infine, comparando le riforme orientate al mercato varate in Gran Bretagna ed in Svezia, Clayton e Pontusson sostengono che «il processo di privatizzazione in Svezia ha riguardato un ambito molto limitato» e che «la direzione del cambiamento nel caso svedese ed in quello inglese è di segno ben diverso; il che

15 Mentre in generale le associazioni di volontariato sono particolarmente numerose nel settore dei servizi socio-assistenziali, in Svezia nell'ambito del volontariato sono largamente dominanti le associazioni culturali e ricreative.

16 I paesi considerati dallo studio sono i seguenti: Svezia, Danimarca, Finlandia, Germania, Olanda, Gran Bretagna e Francia. Per una ricognizione della legislazione e degli interventi nel campo dei servizi alla persona nei paesi dell'Unione Europea si veda anche il rapporto della Commissione Europea intitolato appunto Care in Europe (European Commission 1998). 
suggerisce che i partiti contano ancora (partisan politics still matters)» $(1998,91)$. Mi sembra vi siano buone ragioni per condividere questa affermazione. Sebbene negli ultimi due decenni la scena politica scandinava non sia stata più caratterizzata dall'incontrastato predominio socialdemocratico ed anche i paesi nordici abbiano visto al governo una serie di coalizioni imperniate su partiti conservatori, il loro profilo politico è rimasto comunque radicalmente diverso da quello inglese. Sulla scorta degli elementi presentati in questo articolo, ritengo comunque che questa specifica argomentazione possa essere utilmente integrata da una considerazione dei rapporti inter-governativi e delle differenti dinamiche riscontrabili nei singoli contesti nazionali.

Più precisamente, la forte autonomia impositiva ha consentito agli enti locali scandinavi di salvaguardare il grosso dei servizi pubblici e di impedire qualsiasi drastica inversione di rotta che il governo nazionale avesse eventualmente tentato di imporre per finalità di tipo macro-economico. In altre parole, l'autonomia fiscale ha rappresentato un utile strumento di contrattazione nei confronti del livello centrale, via via che il quadro economico andava deteriorandosi, e grazie ad essa gli enti locali hanno potuto negoziare le misure di austerità partendo da una posizione più forte. Questo vale in misura ancora maggiore in Danimarca ed in Finlandia, dove sono stati istituzionalmente riconosciuti appositi tavoli di contrattazione tra centro e periferia ${ }^{17}$.

In realtà, le ipotesi sviluppate nella letteratura sulla dispersione del potere e sugli effetti connessi alla presenza di una molteplicità di veto points muovono in una diversa direzione. Secondo Huber e Stephens, «le strutture costituzionali che prevedono una pluralità di punti di veto rappresentano un ostacolo alla espansione dell'intervento statale per quanto riguarda il finanziamento e la fornitura pubblica di beni e servizi del welfare state [...] tanto più ambiziosi sono i tentativi di riforma che puntano ad espandere il ruolo pubblico, tanto più vengono colpiti interessi specifici che si trasformano in potenziali oppositori e tanto più grande è il numero di punti di veto nel sistema politico, tanto più ampia sarà la percezione delle probabilità di suc-

17 Blom-Hansen (1998) fornisce una interpretazione in chiave neo-istituzionalista del perché nella dinamica dei rapporti intergovernativi il livello nazionale di governo sia in Svezia che in Norvegia ha svolto un ruolo più incisivo rispetto alla Danimarca e alla Finlandia. 
cesso e dunque la propensione degli oppositori a mobilitarsi» (1996, 13). L'analisi dell'esperienza scandinava suggerisce che a seconda delle circostanze, la presenza di punti di veto può funzionare in maniera opposta. L'esistenza di un elevato numero di punti di veto, così come può bloccare la fornitura pubblica di servizi su vasta scala, può impedirne lo smantellamento nel caso in cui questi siano già in piedi. Le recenti vicende che hanno caratterizzato lo sviluppo delle politiche sociali in Gran Bretagna forniscono a contrario ulteriori elementi di sostegno a questa tesi. Per ragioni di spazio non è possibile diffonderci sul caso inglese; conviene però ricordare almeno che si tratta dell'unico altro paese europeo che, con la Scandinavia, ha precocemente avviato un'ampia rete di servizi territoriali pubblici (anche se non per l'infanzia). Ebbene l'estrema debolezza del governo locale nel sistema politico-amministrativo inglese ed i pesanti limiti di natura giuridica che lo hanno storicamente posto alla mercé del centro (Bulpitt 1983) hanno consentito ai governi conservatori alla guida del paese per diciotto anni consecutivi di ignorare totalmente $i$ tentativi di difesa dei servizi pubblici portati avanti dagli enti locali in mano all'opposizione laburista e di imporre la privatizzazione dei servizi sociali e la loro fornitura in base a criteri di mercato.

\section{Conclusioni}

Se mettiamo a confronto le nostre risultanze con l'interpretazione che viene abitualmente fornita dalla letteratura per descrivere lo sviluppo del governo locale in Europa fino alla metà degli anni settanta, si ha l'impressione di una completa inversione di rotta. Prima degli anni ottanta tutte le ricerche indicavano una crescita progressiva della spesa locale, cui faceva da contraltare una maggiore dipendenza finanziaria degli enti locali dal centro. Esattamente al contrario, gli sviluppi più recenti indicano invece un aumento dell'autonomia fiscale accompagnato questa volta da una incidenza più contenuta della spesa locale sulla spesa pubblica complessiva.

È sullo sfondo di questo quadro istituzionale e finanziario che va considerata la diffusione delle esigenze di care illustrate nella prima parte dell'articolo. Come abbiamo avuto modo di notare, nel corso degli anni ottanta tali nuove esigenze si sono progressivamente diffuse dall'Europa centro-settentrionale a 
quella meridionale e stanno adesso cominciando ad emergere anche nelle zone socialmente ed economicamente più arretrate. Ma l'aumento della domanda di servizi sociali per gli anziani e per l'infanzia, che ha riguardato praticamente tutti i paesi europei, si è inevitabilmente scontrato con uno scenario segnato da confining conditions decisamente avverse.

Le argomentazioni sviluppate nel corso di questo lavoro avvalorano l'importanza critica del timing nello sviluppo di questa specifica area di policy. Innanzitutto, a seconda della particolare giuntura storica, i decisori pubblici sono chiamati a misurarsi con vincoli ed opportunità economiche di segno diverso. Nella fattispecie, a differenza di quanto è avvenuto nei paesi scandinavi, la pressione fiscale complessiva aveva ormai raggiunto il limite massimo quando si è posta la questione dei servizi sociali nel resto dell'Europa. In un contesto di questo tipo, qualsiasi politica espansiva era sostanzialmente impraticabile ed i diversi livelli di governo sono stati costretti a contendersi la stessa fetta della torta. Ma non è solo una questione di opportunità economiche. A seconda del timing, variano anche le possibili interconnessioni tra ambiti sociali e/o politici frutto di distinte sequenze causali. Il diverso intersecarsi di tali processi, in funzione dello specifico momento congiunturale, produce da un lato un coacervo di effetti collaterali e conseguenze impreviste che varieranno appunto a seconda dei momenti temporali e dall'altro condiziona significativamente l'arco delle alternative di policy disponibili. La specifica interconnessione che si è venuta a determinare nel caso della Francia tra le problematiche relative alla disoccupazione e la questione di servizi sociali è particolarmente istruttiva a questo proposito.

Tirando le fila del discorso, possiamo dire che era alquanto improbabile che i paesi dell'Europa continentale e meridionale seguissero le orme del modello scandinavo. Quel particolare percorso non era più disponibile in una fase temporale successiva. Ciò nondimeno, gli attuali sforzi appaiono decisamente insufficienti alla luce dei mutamenti sociali in corso e ancor più delle prospettive che ci attendono nell'immediato futuro. In sintonia con tale valutazione pessimistica, è lecito attendersi che l'ideale di una «cittadinanza inclusiva» (Knijn e Kremer 1997) capace cioè di tener conto dei bisogni di care che vanno accentuandosi sempre più nel tessuto sociale dei paesi occidentali sia destinato ad essere assai più oggetto di dibattito accademico che non di traduzione operativa. Tuttavia, in questo panorama 
nell'insieme assai poco roseo, possiamo aspettarci a livello locale anche delle traiettorie divergenti. Da una molteplicità di segnali si può infatti desumere come il processo di standardizzazione, che ha segnato lo sviluppo del welfare state europeo dal 1945 alla metà degli anni settanta, stia progressivamente cedendo il passo ad una crescente variabilità delle politiche sociali a livello sub-nazionale.

\section{Riferimenti bibliografici}

Antonnen, A. e J. Sipilä (1996), European Social Care Services: Is it Possible to Identify Models?, in «Journal of European Social Policy», n. 2, pp. 87-100.

Alber, J. (1995), A Framework for the Comparative Study of Social Services, in «Journal of European Social Policy», n. 2, pp. 131-149.

Ascoli, U. (a cura di) (1999), Il Welfare futuro: manuale critico del Terzo settore, Roma, Carocci.

Bennet, R. (1993), Local Government in the New Europe, London, Bellhaven Press.

Blom-Hansen, J. (1998), Macroeconomic Control of Local Governments in Scandinavia: The Formative Years, in «Scandinavian Political Studies», n. 2, pp. 129-159.

Bulpitt, J. (1983), Territory and Power in the United Kingdom, Manchester, Manchester University Press.

Bussemaker, J. (1998), Rationales of Care in Contemporary Welfare States: The Case of Childcare in the Netherlands, in «Social Politics», n. 2, pp. 70-96.

Chuliá, E. e B. Alvarez-Miranda (1999), Pautas Presentes y Tendencias Futuras en la Prestación de Cuidados a Mayores en España. ¿Hacia un Reequilibrio de la Contribución de Estado, Empresas y Familias?, relazione presentata al quarto congresso della Associazione di Scienza Politica Spagnola, Granada, Settembre.

Clayton, R. e J. Pontusson (1998), Welfare State Retrenchment Revisited. Entitlements Cuts, Public Sector Restructuring, and In-egalitarian Trends in Advanced Capitalist Societies, in «World Politics», n. 1, pp. 67-98.

Commissione Europea (1997), Relazione Demografica, Luxembourg.

Council of Europe (1975), The Financial Structures of Local and Regional Authorities in Europe, vol. I, Strasbourg.

Dente, B. e F. Kjellberg (1988), The Dynamis of Institutional Change, London, Sage.

European Commission (1998), Care in Europe - Joint Report of the «Gender and Employment» and «Gender and Law» groups of experts, Luxembourg. 
Eurostat (anni vari), Statistiques en Bref.

Fargion V. (1997a), Current Social Service Regimes in Europe: The Rise and Development (1965-1995), relazione presentata al convegno annuale Isa RC 19, Copenhagen, agosto.

- (1997b), Geografia della cittadinanza sociale in Italia, Bologna, Il Mulino.

Ferrera, M. (1993), Modelli di solidarietà, Bologna, Il Mulino.

Huber, E. e J. Stephens (1996), Political Power and Gender in the Making of the Social Democratic Service State, relazione presentata al convegno annuale Isa RC 19, Canberra, agosto.

Knijn, T. e M. Kremer (1997), Gender and the Caring Dimension of Welfare States: Toward Inclusive Citizenship, in «Social Politics», n. 3, pp. 328-361.

Kramer, R., H. Lorentzen, W. Melief e S. Pasquinelli (1993), Privatisation in Four European Countries: Comparative Studies in Government-Third Sector Relationships, New York, Sharpe.

Kröger, T. (1997), Local Government in Scandinavia: Autonomous or Integrated into the Welfare State, in Sipilä (a cura di), pp. 95-107.

Jeppsson Grassman, E. (1998), The Voluntary Sector in a Welfare Perspective, relazione presentata alla conferenza «Comparing Social Welfare Systems in Nordic Countries and France», Gilleleje, settembre.

Laville, J.L. (1996), Services de proximité: la construction sociale d'un champ d'activités economiques, relazione presentata alla conferenza «Terzo settore, stato e mercato nella trasformazione delle politiche sociali in Europa», Milano, Università Cattolica, ottobre.

Martin, C. (1999), Reframing Social Policies in France towards Selectivity and Commodification: The Case of Family and Frail Elderly Policies, relazione presentata alla conferenza su «Reforming Social Assistance and Social Services: International Experiences and Perspectives», Firenze, Istituto Universitario Europeo, dicembre.

Mire (Mission de recherche et expérimentation) (1996), Comparer les systémes de protection sociale en Europe, vol. 2, Paris, Imprimerie Nationale.

- (1998), Social Protection: A Bilingual Glossary, vol. 1, Paris, Imprimerie Nationale.

Moreno, L. e A. Arriba (1999), Decentralization and the New Logic of Welfare Provision in Spain, relazione presentata alla conferenza su «Reforming Social Assistance and Social Services: International Experiences and Perspectives», Firenze, Istituto Universitario Europeo, dicembre.

Ocse (1996), Caring for Frail Elderly People. Policies in Evolution, Paris. Page, E. (1987), Central and Local Government Relations. A Comparative Analysis of West European Unitary States, London, Sage.

Palier, B. (1998), La référence au territoire dans les nouvelles politiques sociales, in «Politiques et Management Public», n. 3, pp. 13-41. 
Pierson, P. (1999), Not just What, but When: Issue of Timing and Sequence, Istituto Universitario Europeo, maggio, di prossima pubblicazione in «Studies in American Political Development».

Ranci, C. (1999), Oltre il Welfare State. Terzo settore, nuove solidarietà e trasformazioni del welfare, Bologna, Il Mulino.

Rostgaard, T. e T. Fridberg, (1998), Caring for Children and Older People. A comparison of European Policies and Practices, Copenhagen, The Danish National Institute of Social Research.

Sipilä, J. (a cura di) (1997), Social Care Services: The Key to the Scandinavian Welfare Model, Aldershot, Ashgate.

Schultheis, F. (1996), La famille, une catégorie du droit social? Une comparaison franco-allemande, in Mire (1996), pp. 203-234.

Trägårdh, L. (1997), Statist Individualism: On the Culturality of the Nordic Welfare State, in O. Soresen e B. Sträth (a cura di), The Cultural Construction of Norden, Oslo, Scandinavian University Press, pp. 253-285.

Trifiletti, R. (1998), Restructuring Social Care in Italy, in J. Lewis (a cura di), Gender, Social Care and Welfare State Restructuring in Europe, Aldershot, Ashgate, pp. 175-206.

Van Kersbergen, K. (1995), Social capitalism, London, Routledge.

Windebank, J. (1996), To What Extent Can Social Policy Challenge the Dominant Ideology of Mothering? A Cross-national Comparison of Sweden, France and Britain, in «Journal of European Social Policy», n. 2, pp. 147-161. 